University of Louisville

ThinkIR: The University of Louisville's Institutional Repository

Electronic Theses and Dissertations

8-2005

\title{
Interpersonal change as an outcome of time-limited interpersonal therapy.
}

Nicholas Lawrence Salsman 1978-

University of Louisville

Follow this and additional works at: https://ir.library.louisville.edu/etd

\section{Recommended Citation}

Salsman, Nicholas Lawrence 1978-, "Interpersonal change as an outcome of time-limited interpersonal therapy." (2005). Electronic Theses and Dissertations. Paper 1256.

https://doi.org/10.18297/etd/1256

This Doctoral Dissertation is brought to you for free and open access by ThinkIR: The University of Louisville's Institutional Repository. It has been accepted for inclusion in Electronic Theses and Dissertations by an authorized administrator of ThinkIR: The University of Louisville's Institutional Repository. This title appears here courtesy of the author, who has retained all other copyrights. For more information, please contact thinkir@louisville.edu. 
INTERPERSONAL CHANGE AS AN OUTCOME OF TIME-LIMITED INTERPERSONAL THERAPY

\author{
By \\ Nicholas Lawrence Salsman \\ B.A., Transylvania University, 2000 \\ M.A., University of Louisville, 2003

\begin{abstract}
A Dissertation
Submitted to the Faculty of the Graduate School of the University of Louisville in Partial Fulfillment of the Requirements for the Degree of
\end{abstract} \\ Doctor of Philosophy \\ Department of Psychological and Brain Sciences \\ University of Louisville \\ Louisville, Kentucky
}

August 2005 

Copyright 2005 by Nicholas L. Salsman

All rights reserved 
INTERPERSONAL CHANGE AS AN OUTCOME OF TIME-LIMITED INTERPERSONAL THERAPY

By

Nicholas Lawrence Salsman

B.A., Transylvania University, 2000

M.A., University of Louisville, 2003

A Dissertation Approved on

June 16, 2005

by the following Dissertation Committee:

Dissertation Director 


\section{ACKNOWLEDGEMENTS}

I dedicate this dissertation to my wife, Tiffany. She has been an incredible and constant source of support, motivation, and wisdom throughout this process. I am grateful to my mother, Jane Salsman, for all of the sacrifices she made so that I can be where I am today. I would like to thank my mentor, Dr. Stan Murrell, for all of his guidance and for providing an exemplary model of who I strive to be in my own career. I express gratitude to my committee members, Dr. Rich Lewine, Dr. Paul Salmon, Dr. Wayne Usui, and Dr. Janet Woodruff-Borden, for their time, kindness, and expertise in shaping not only this project, but also my professional development. I also extend my appreciation to Dr. Tracy Eells and Dr. Bernadette Walter for their ever-present willingness to help. Finally, I would like to thank my friends and family whose love and support has carried me through the challenges I have faced. I am especially grateful to my brothers, Sammy and Brandon Salsman; my grandmother, the late Clarice Spalding; the late Alvin Beckley; and the late Dr. Arma Peltier. 


\section{ABSTRACT \\ INTERPERSONAL CHANGE AS AN OUTCOME OF TIME-LIMITED \\ INTERPERSONAL THERAPY}

Nicholas L. Salsman

June 16, 2005

This study examined two questions related to interpersonal change as an outcome of Time-Limited Interpersonal Therapy (TLIPT). The first asked if clients experience both symptom change and interpersonal change when treated with TLIPT. The second explored factors that contribute to interpersonal changes in clients treated with TLIPT. The study examined a sample of 61 clients who identified interpersonal problems as a primary therapeutic issue and were treated with between 9 and 76 sessions of TLIPT. Repeated measures analysis of variance (ANOVA) of the first question revealed that clients experienced significant reductions in measures of symptoms and interpersonal distress. Clients also experienced significant increases in therapist-rated interpersonal affiliation dimension (i.e., clients increased in friendliness and decreased in hostility) and self-rated interpersonal control (i.e., clients increased in dominance and decreased in submissiveness). Additionally, statistical trends suggested that clients experienced modest reductions in the self-rated and therapist-rated measures of interpersonal rigidity and extremity; modest increases in self-rated and significant other-rated affiliation; and modest increases in therapist-rated control. Analyses of the second question explored the role of early alliance, early symptom reduction, early stage and middle stage therapist and 
client complementarity in contributing to interpersonal change. ANOVA comparisons of those with high interpersonal changes and those with low interpersonal changes indicated that there were no statistically significant differences in these variables between the two groups. However, statistical trends indicate that higher early complementarity on the control dimension may be associated with reductions in interpersonal rigidity and increases on the control dimension; and that higher affiliation complementarity in the middle of therapy may be associated with increases on the affiliation dimension. Further analyses revealed that therapists and clients had significantly lower complementarity on the affiliation dimension in the middle phase of therapy than in the early phase of therapy. Implications for future research are discussed. 
TABLE OF CONTENTS

PAGE

ACKNOWLEDGMENTS............................................................

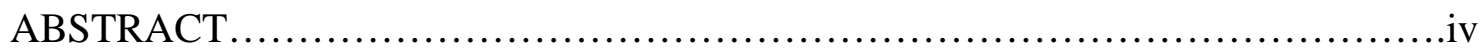

LIST OF TABLES ...............................................................

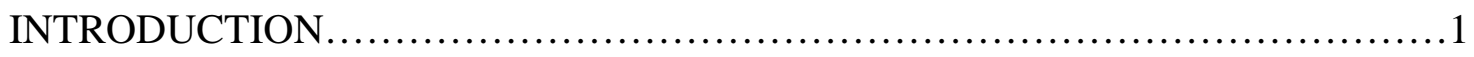

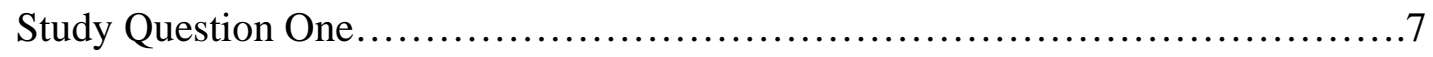

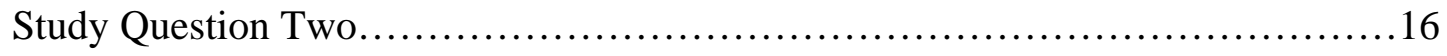

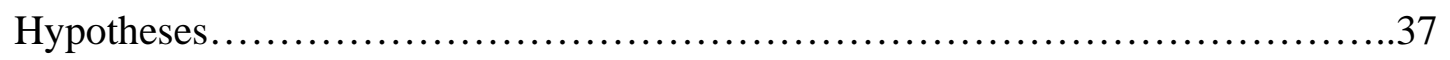

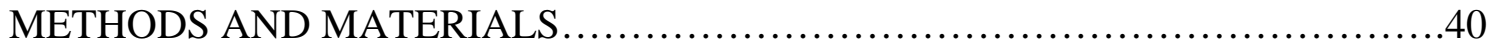

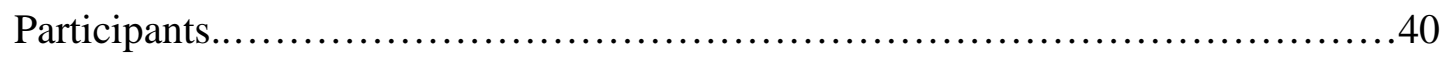

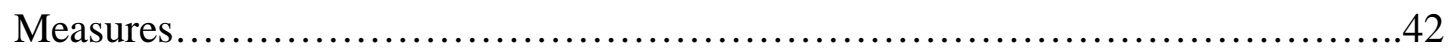

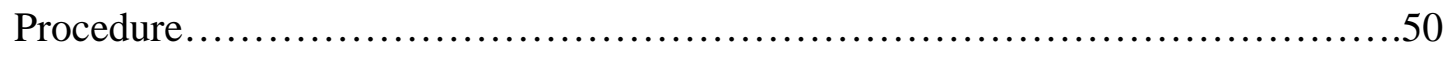

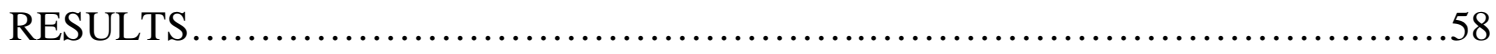

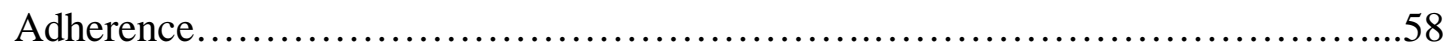

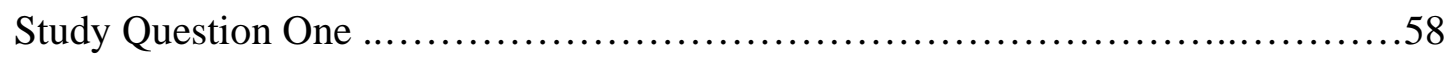

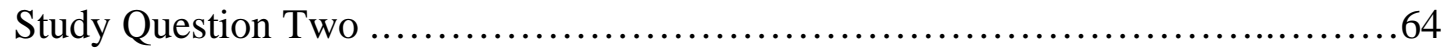

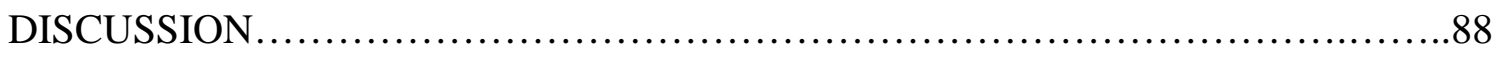

SUMMARY AND CONCLUSIONS.............................................101

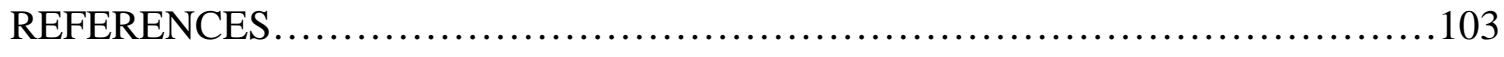

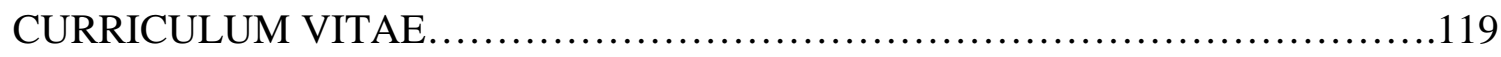




\section{LIST OF TABLES}

TABLE

PAGE

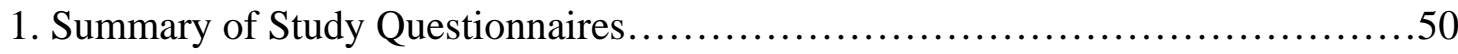

2. Descriptive Statistics for Outcome Variables ................................60

3. Summary of Results for Study Question One.................................65

4. Means of Vector Length Variables among K-Means Vector Length Clusters .......75

5. Means of Therapy Process Variables among K-Means Vector Length Clusters.... .77

6. Means of Affiliation Variables among K-Means Affiliation Clusters ...............79

7. Means of Therapy Process Variables among K-Means Affiliation Clusters .........81

8. Means of Control Variables among K-Means Control Clusters .....................83

9. Means of Therapy Process Variables among K-Means Control Clusters............85

10. Summary of Results for Study Question Two.................................86 


\section{INTRODUCTION}

This study sought to understand the process of bringing about changes in maladaptive interpersonal patterns through psychotherapy for the treatment of interpersonal problems, i.e., Time-Limited Interpersonal Therapy (TLIPT, Murrell, 2003). Theories of psychotherapy guide interventions in order to bring about changes in the theorized underlying cause of psychological problems, e.g., emotional re-learning within the context of the therapeutic relationship for maladaptive interpersonal patterns, medication for brain chemistry, behavioral modification for learned behaviors, cognitive restructuring for cognitive schemas, etc. Little research has addressed interpersonal change as an outcome of psychotherapy. This study sought to increase research in this area and addressed two questions regarding interpersonal change as an outcome of TLIPT. The first, do clients experience both symptom change and interpersonal change when treated with TLIPT? Research has suggested that TLIPT would produce symptom reductions, reductions in measures of interpersonal distress, and changes in interpersonal patterns. The second, what factors contribute to interpersonal changes in clients treated with TLIPT? Research and theory have suggested that elements such as initial complementarity between the therapist and the client, higher levels of early working

alliance, and early symptom reductions would lead to interpersonal change. Research and theory have also suggested that following the beginning phase of treatment, the therapist's provision of responses that are non-complementarity to the client's 
maladaptive pattern in the middle phase of treatment would also be associated with interpersonal change.

The Development of Maladaptive Interpersonal Patterns: Infancy through Adulthood

The theoretical underpinnings of Murrell’s (2003) TLIPT approach to therapy are based on what may be described as an interpersonal learning theory. This theory describes how individuals learn ways of relating to others beginning with, perhaps most importantly, the infant/caregiver relationship. TLIPT's understanding of the development of interpersonal patterns is based on the interpersonal theory of Sullivan (1953a), and refinements by Leary (1957), Carson (1969), and Kiesler (1996) and is heavily rooted in the attachment theory developed by Bowlby (1988).

Bowlby (1988) has asserted that humans have a primary motivation to relate to others in order to avoid threats to survival. Relatedness for infants is a matter of survival (i.e., without a relationship to a caregiver, the infant will be unable to care for him or herself). Thus, infants learn patterns of relating that are essential to survival. Deviation from this learned pattern can lead to survival anxiety and/or threats to survival and is therefore avoided. An infant learns a pattern of interaction with his or her primary caregiver that is the most effective path for the infant to obtain what is necessary for survival and avoid what threatens survival. The infant learns to engage in this interpersonal pattern in order to obtain the relatedness necessary to avoid survival anxiety. A tender, caring, healthy infant/caregiver dyad can engender a sense of security and positive self-esteem in the infant, while other less healthy patterns can lead to maladaptive patterns. Continual disapproval of the infant's needs by the caregiver, for example, could lead the infant to learn a pattern of being nonassertive and avoiding the 
expression of needs. This submissive pattern is reinforced by the infant's avoidance of abandonment and subsequent survival anxiety. Based on the rigidity and extremity of the caregiver's disapproving stance with the infant, the infant will develop a correspondingly rigid and extreme interpersonal pattern. In other words, the more a caregiver adheres to a particular pattern across situations, the more likely it is that the infant will tend to adhere to the complementary pattern across situations. Bowlby's research has supported that interactions beginning in infancy contribute to adaptive and maladaptive interpersonal characteristics (e.g., Bowlby, 1970)

Interpersonal patterns are constantly subjected to new learning experiences. The base pattern developed in infancy is adjusted through interactions with others throughout life. For instance, Sullivan (1953a) speaks of the importance of a "chum" in adolescence. This chum relationship is used to test out and further develop the principles of trust with a same age peer. A body of research has also examined the importance of adult relationships on the maintenance and refinement of interpersonal attachment patterns (e.g., Simpson \& Rholes, 1998). While a person’s interpersonal pattern may be adjusted based on interactions with others, as the rigidity and extremity of the person's pattern increase, so to do the chances that a person may elicit a response that reinforces their current pattern, and therefore resist adjustment. Dietzel and Abeles (1975) found that more maladjusted patients had a higher degree of success in eliciting complementary reactions from their therapists throughout therapy than less maladjusted clients. Nelson (1984) found that both psychiatric inpatients and non-patients who interacted with confederates who rigidly adhered to a particular interpersonal style tended to respond in complementary ways to the confederates. Thus, research supports that rigid and extreme 
interpersonal behavior can often lead to others providing responses that reinforce the rigid and extreme interpersonal behavior. Van Denburg and Kiesler (1993) further clarified the role of rigidity and extremity through their finding that individuals' rigidity and extremity increased as interview stress increased. Thus, as stress increases, people may more rigidly adhere to their most comfortable and familiar interpersonal behaviors. Rigid and extreme patterns serve an adaptive function of avoiding further arousal of severe anxiety (i.e., the anxiety that comes about through behaving in a manner that is inconsistent with one's typical interpersonal style) and therefore may lead people to sacrifice relatedness for the purpose of warding off this anxiety. Thus, while engaging in a small set of extreme behaviors across interpersonal situations (e.g., extreme shyness) will likely lead to poor interpersonal relationships, it may also serve the purpose of avoidance of anxiety.

An assumption of the TLIPT approach to therapy is that maladaptive interpersonal patterns lead to presentations of symptoms and problems such as depression, anxiety, low self-esteem, lack of social skills, difficulty with adjustment, etc. Therapy clients may hold maladaptive internal models of the self, of others, or perhaps most likely, an interaction of both (Bowlby, 1988). In other words, clients may have a set of beliefs about themselves and others in interpersonal interactions, which lead them to behave in maladaptive ways. The task of therapy is to alleviate these problems and symptoms by working to help the client develop a more adaptive interpersonal pattern.

\section{Conceptualization of Interpersonal Patterns}

Interpersonal theory has come to conceptualize interpersonal patterns in terms of two orthogonal dimensions. The first dimension is a measure of interpersonal closeness 
called affiliation. It has polar ends of friendly and hostile. The second dimension is interpersonal control, with polar ends of dominant and submissive. These orthogonal dimensions form two axes, perpendicular to one another with dominant at the top, hostile at the left, submissive at the bottom, and friendly to the right. A circle can be transposed on these axes to form an interpersonal circumplex. Various circumplexes have been described in the literature (e.g., the Inventory of Interpersonal Problems - Circumplex (IIP) described by Alden, Wiggins, and Pincus, 1990; the Impact Message Inventory (IMI) circumplex described by Kiesler, 1987; the Interpersonal Checklist circumplex described by Leary, 1957; and the Interpersonal Adjectives Scale circumplex described by Wiggins, Trapnell, \& Phillips, 1988), but a commonly used structure consists of the eight descriptors using the axes or combinations of the axes as labels (i.e.,hostiledominant, hostile, friendly-submissive, etc.). Figure 1 provides an example of a typical interpersonal circumplex format.

Interpersonal circumplex patterns can be used to describe a person’s typical interpersonal style. Elements of a person's pattern include the degree of a person’s affiliation (i.e., high, positive scores represent a high degree of friendliness and low, negative scores represent a high degree of hostility), control (i.e., high, positive scores represent a high degree of dominance and low, negative scores represent a high degree of submissiveness), and rigidity and extremity (i.e., high scores represent a rigid and extreme interpersonal pattern and low scores represent a flexible and moderated approach to interpersonal interactions). Interpersonal measures have been developed to describe an individual's interpersonal pattern from their own self-report (e.g., the Inventory of Interpersonal Problems, IIP, Horowitz, Rosenberg, Baer, Ureno, \& Villasenor, 1988) or 


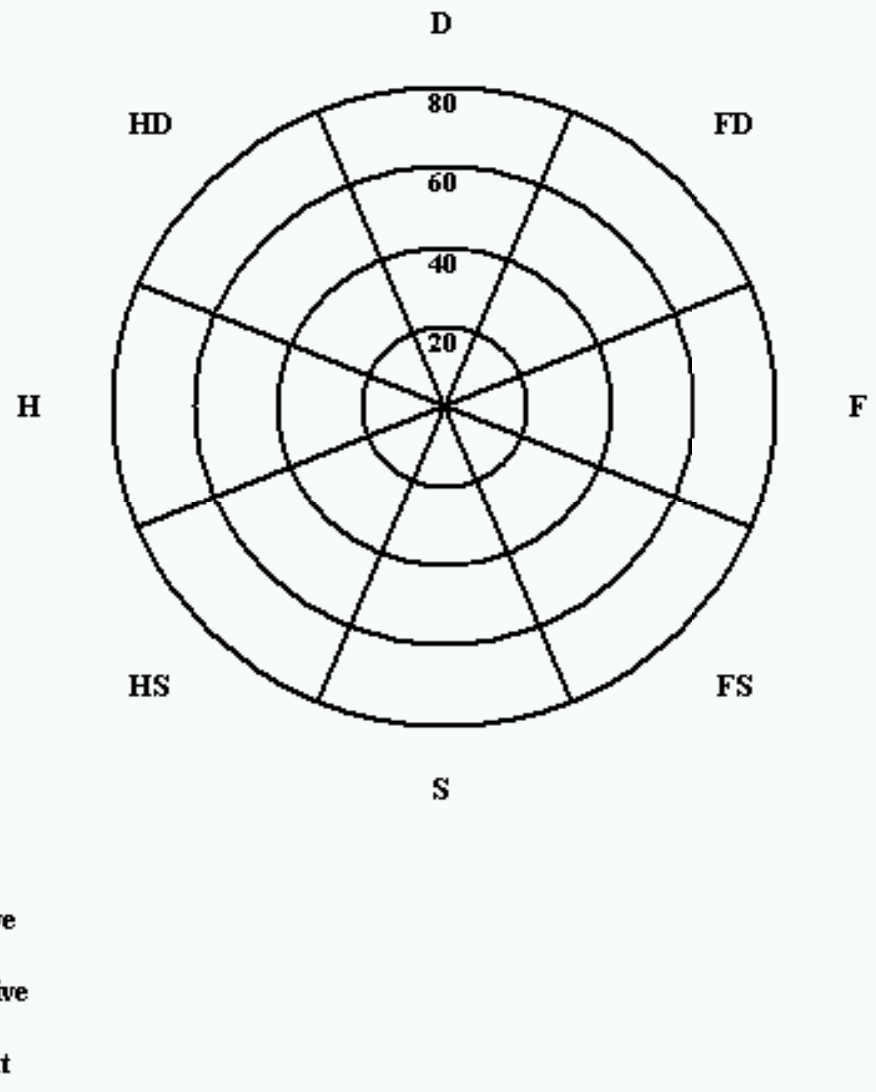

Figure 1. A basic interpersonal circumplex. 
from another person’s perspective (e.g., the Impact Message Inventory, IMI, Kiesler \& Schmidt, 1993). Interpersonal circumplex measures were used in this study to measure changes in clients' interpersonal patterns over the course of therapy.

Study Question One: Do clients experience both symptom change and interpersonal change when treated with TLIPT?

\section{Interpersonal Changes and Symptom Changes as Psychotherapy Outcomes}

Research has supported that both interpersonal pattern changes and symptom reductions are related and important outcomes of psychotherapy. While interpersonal measures and more symptom-based outcomes are often significantly correlated, they are not completely overlapping constructs. For example, Lambert et al. (1996) found a correlation of -.56 between a symptom-based measure and interpersonal distress. That is, measures of interpersonal patterns appear to examine some overlapping constructs with symptom measures and some constructs that extend beyond typical symptom measures. Support for this is found in a study by Horowitz et al. (1988), which indicated that while clients' symptom reductions tended to stabilize at session ten, their interpersonal distress continued to decrease beyond session ten. Svartberg, Stiles, and Seltzer (2004) compared outcomes for patients treated with cognitive-behavioral therapy (CBT) and dynamic therapy for Cluster C personality disorders. They found that patients treated in both conditions had reductions in symptoms and reductions in interpersonal distress. In a study of individuals with personality disorders who were first treated with day treatment and subsequently treated with group therapy, Wilberg et al. (2003) found reductions in both symptoms and interpersonal problems as measured by the IIP. Rice (2003) found that individuals in group therapy tended to have improvements in both interpersonal 
problems and symptoms. Thus, both symptoms and interpersonal change appear to be important outcomes across a variety of therapy modalities with a variety of clients.

Research has indicated that symptoms and interpersonal change appear to be interrelated. That is, decreases in symptoms and changes in maladaptive interpersonal patterns affect one another. Borkovec, Newman, Pincus, and Lytel (2002) found that in a sample of anxious clients treated with CBT, higher interpersonal problems measured at the end of therapy were associated with worse symptom-focused outcomes in the followup period after therapy. That is, clients with more interpersonal problems at the completion of therapy tended to do worse after therapy than those with fewer interpersonal problems. Wagner (2003) studied a sample of clients in processexperiential psychotherapy. He found that the changes in measures of symptoms were significantly correlated with changes in interpersonal problems. Wagner (2003) highlights the importance of examining change in psychotherapy using a variety of measures including measures of symptoms and interpersonal change. It appears that both symptom reduction and improved interpersonal functioning are both important components of recovery in psychotherapy. Further, interpersonal change may be an important contributor to post-therapy outcomes. Therefore, this study utilized measures of both symptom change and interpersonal change as outcomes of TLIPT.

Previous studies of clients in therapies similar to TLIPT (i.e., therapies focused on interpersonal problems) have supported that therapy leads to significant interpersonal changes. Connolly et al. (1999) compared outcomes of a supportive-expressive therapy that focused on interpersonal pattern change with medication for clients with Generalized Anxiety Disorder. They found significant improvement of recognition of interpersonal 
problems, decrease of interpersonal distress, and a decrease in symptoms in clients treated with the interpersonally focused therapy. Additionally, the authors found that those treated with interpersonally focused therapy had significant changes in their selfunderstanding of their own patterns of interpersonal wishes, changed typical responses in interpersonal situations, and altered typical responses of others to the person in interpersonal situations. They found that the clients treated with medication improved only on symptom measures and recognition of interpersonal problems, but did not have significant changes in their self-understanding of their patterns of interpersonal wishes, in their typical responses in interpersonal situations, or in the typical responses of others. Thus, those treated with pharmacotherapy did not appear to have changes in their interpersonal interactional style. Those treated with psychotherapy had a significantly greater increase in self-understanding than those treated with medication with an effect size measured by Cohen's $d$ of .67, which is in the medium to large range. This provides evidence that psychotherapy focused on changing interpersonal patterns can bring about interpersonal changes, which extend beyond the outcomes of a therapy that does not focus on these elements such as pharmacotherapy.

Interpersonal change appears to be an important outcome in a number of types of therapy. As described above, studies have found that interpersonal change occurs in numerous types of therapy, for example, cognitive-behavioral therapy and dynamic therapy (Svartberg et al., 2004), day treatment and group therapy (Wilberg et al., 2003), and process experiential therapy (Wagner, 2003). In her review, Connolly Gibbons (2004) found that interpersonal content was an important and proportionally high area of focus in psychodynamic, cognitive, and interpersonal therapies. The interpersonal focus 
in these therapies is often on present day relationships, rather than past. This study of interpersonal change in TLIPT may provide further insight to interpersonal change as it occurs in interpersonal therapy.

Research has indicated that while there is consistency in interpersonal patterns across different individuals, interpersonal patterns can and do vary depending on the relationships where they are applied (e.g., a person may have different interpersonal tendencies with the boss than with a spouse). Connolly et al. (1996) utilized cluster analytic methods for a sample of clients and found that individuals typically had between one and five distinct interpersonal patterns with individuals in their lives, with a main cluster typically representing the most pervasive interpersonal pattern. They found that $60 \%$ of the interpersonal patterns identified with the therapist were significantly correlated with one of the five interpersonal patterns with other individuals in their lives. The most pervasive of the patterns was utilized with the therapist in $34 \%$ of the total sample. Connolly, Crits-Christoph, Barber, \& Luborsky (2000) replicated this study with a new sample and found that individuals had between one and three distinct interpersonal patterns, with a main cluster representing the most pervasive interpersonal pattern. In this sample, $44 \%$ of interpersonal patterns identified in the therapeutic relationship significantly correlated with one of the three interpersonal patterns found with others. The most pervasive pattern was utilized with the therapist in 33\% of the total sample. Thus, it appears that the interpersonal pattern enacted with the therapist may often represent the person's most pervasive interpersonal pattern outside of therapy. However, it also may be that the interpersonal approach of the client within therapy is distinct from interpersonal patterns outside of therapy. Therefore, for this study it was important to 
look at interpersonal changes measured from different perspectives, i.e., examining clients' interpersonal patterns within different relationships and from different perspectives using a multi-rater, multi-measure approach. This study examined changes that may occur in multiple domains including clients' relationships with the therapist, a significant other, and clients’ self-reported general interpersonal style. This study also examined different areas of change including symptom reduction, interpersonal distress, affiliation dimension, control dimension, and rigidity and extremity changes.

\section{Symptom Reduction}

Numerous studies have examined symptom reduction as an outcome of psychotherapy. One of the most prominent recent studies of the effectiveness of psychotherapy in producing symptom reductions is the NIMH Collaborative Treatment of Depression Study (Elkin et al., 1989). The authors compared 239 clients randomly assigned to one of four treatment conditions: interpersonal psychotherapy, CBT, pharmacotherapy with imipramine, or placebo. The authors found that both forms of psychotherapy and imipramine were effective in reducing symptoms.

Numerous other sources review psychotherapy research and show that psychotherapy tends to lead to symptom reduction and overall improvements. More generally, a study by Consumer Reports magazine (1995) surveyed over 4,000 of their readers who had been in psychotherapy. The survey found that nearly $90 \%$ of respondents reported significant improvements in their mental health after psychotherapy. This study has often been cited as an unbiased (i.e., not effected by researcher or funding allegiance) indication of the effectiveness of psychotherapy. Numerous studies have also examined the effectiveness of psychotherapy through meta-analysis. In their review of 
meta-analyses, Asay and Lambert (1999) found that the average effect size of psychotherapy is 0.82 , compared to an effect size of 0.42 for minimal treatment/placebo and an effect size of 0.00 for no treatment/control. Kiesler (1996) specifically reviewed much of the research on symptom reductions in interpersonal psychotherapy and found that generally interpersonal therapy tends to be effective in producing symptom reductions. Symptom reductions were examined as indicators of positive outcome in TLIPT in this study.

\section{Interpersonal Distress}

Interpersonal distress is a measure of overall interpersonal problems that is calculated as the mean of all items on the IIP (Horowitz et al., 1988). Horowitz et al. found that clients' pretreatment scores correlated $(r=.64)$ with total scores on the Symptom Checklist-90-Revised (Derogatis, 1977) and with post-treatment scores ( $r=$ .57). This indicated that while interpersonal distress has some similarities to symptoms as outcomes, interpersonal distress appears to have unique variance as a measure of outcome. The current study examined changes in interpersonal distress as indication of improvement of interpersonal outcomes that go beyond symptom reductions.

Research has indicated that interpersonal distress tends to reduce over the course of psychotherapy. Horowitz et al. (1988) found that clients treated with psychotherapy tended to have reductions in distress. Rosenthal, Muran, Pinsker, Hellerstein, and Winston (1999) studied outcomes of 12 clients treated with a brief supportive therapy model similar to the TLIPT approach. They found that clients' IIP distress score significantly reduced over the course of 40 sessions. The current study tested if clients in TLIPT experienced reductions in interpersonal distress. 
While IIP distress certainly represents one level of interpersonal outcome, namely the amount of interpersonal problems, this measure does not capture change in the control dimension, affiliation dimension, or interpersonal rigidity and extremity. Connolly et al. (1999) found that several measures of interpersonal patterns were not significantly related to IIP interpersonal distress. The authors indicate that while IIP distress may measure general interpersonal problems, other measures are necessary to examine interpersonal pattern change. Therefore, in addition to changes in IIP distress, the current study examines changes in measures of control, affiliation, and rigidity and extremity. Interpersonal Rigidity and Extremity

Some research has also examined changes in interpersonal rigidity and extremity as indicators of psychotherapy outcome. Kiesler (1983) stated that successful psychotherapy clients would show reductions in rigid and extreme interpersonal behavior. Kivlighan, McGovern, and Corazzini (1984) found that clients in a study of group therapy tended to decrease the amount they rigidly adhered to their peak interpersonal style over the course of therapy. Rosenthal et al. (1999) presented IIP circumplex data on four individuals suggesting change in interpersonal patterns, with the individuals showing consistent reductions in rigidity and extremity of their interpersonal patterns. The authors called for research with larger samples and with additional interpersonal measures to further support that therapy of this nature can bring about changes in interpersonal patterns in addition to symptom reductions. This study attempted to answer this call by systematically measuring interpersonal change in a larger sample with a multi-measure, multi-rater approach. 
Interpersonal rigidity and extremity have also been linked to a number of other outcomes and variables associated with outcome. Ruiz et al. (2004) found that higher interpersonal rigidity was negatively associated with outcomes at the end of treatment in a naturalistic sample of community therapy clients. Kiesler and Watkins (1989) found that higher extremity of client's interpersonal hostility was significantly related to lower client rated alliance and that higher extremity of client's total, hostile, and friendly interpersonal behavior was significantly related to lower therapist rated alliance. Thus, the more extreme the client's hostility, the weaker the client perceived the alliance and the more extreme the client's overall interpersonal behaviors, the weaker the therapist perceived alliance. This study examined changes in rigidity and extremity as outcomes of TLIPT.

\section{The Affiliation Dimension}

Research on overall changes in the interpersonal affiliation dimension over the course of psychotherapy has been limited. However, those studies that have examined affiliation changes in psychotherapy have been relatively consistent in findings indicating that clients tend to become friendlier and less hostile over the course of therapy. Granberg and Armelius (2003) found that a sample of severely disturbed psychiatric patients, on average, experienced increased affiliation over the course of five years of milieu treatment. Constantino's (2003) results indicated that clients in CBT for generalized anxiety disorder had increases in affiliation over the course of therapy. Sledge (1999) found that depressed clients treated with CBT tended to develop increased interpersonal friendliness over the course of therapy. Finally, in a case study, McCullough and Carr (1987) indicated that the client experienced an increase in 
friendliness over the course of therapy. Thus, findings indicate that among a variety of different types of treatment modalities, clients tended to experience increases in interpersonal affiliation.

Further research has indicated that increases in affiliation are linked with other positive processes and outcomes. Malcolm (2000) found that compared to those who did not resolve unfinished business in process-experiential therapy, those who did resolve unfinished business (i.e., the goal of this type of process-experiential therapy) had greater increases in affiliation. McMain's (1996) results indicated that increases in affiliation in clients treated with process-experiential therapy were associated with better symptom outcomes. Thus, increase in affiliation appears to be an important outcome. This study examined if TLIPT clients tend to experience increased affiliation over the course of therapy.

\section{The Control Dimension}

Research on overall changes in the interpersonal control dimension over the course of psychotherapy has also been limited. Again, the limited research has been consistent, in this case, showing that clients generally tend to experience reductions in submissiveness and increases in dominance over the course of therapy. Sledge (1999) studied a sample of chronically depressed community outpatients treated with CBT specifically intended for the chronically depressed. The author found that the outpatients' interpersonal patterns tended to shift from more submissive styles to more dominant styles. Filak, Abeles, and Norquist's (1986) results indicated that among successful clients receiving an average of 24 individual therapy sessions, a significant number showed a shift in their interpersonal pattern from submissive at pretherapy to 
dominant at post therapy. Werner (1984) found that spouses, friends, and relatives of clients in psychotherapy rated decreases in submissiveness over the course of therapy. In a case study, McCullough and Carr (1987) indicated that the client developed increased dominance over the course of therapy. Thus, this study examined if TLIPT clients show shifts in their control scores toward more dominance and less submissiveness.

Study Question Two: What factors contribute to interpersonal changes in clients treated with TLIPT?

In addition to exploring the interpersonal changes that occur in a sample of clients treated with TLIPT, this study will also explore factors that contribute to interpersonal changes. Previous research has identified several factors that are important contributors of symptom reductions and show promise as predictors of interpersonal change. These factors include interpersonal complementarity, therapeutic alliance, and early symptom reductions. This study explores if these factors do in fact contribute to interpersonal change.

\section{Interpersonal Complementarity}

Interpersonal theory predicts that in a two-person interaction, one person's interpersonal style exerts pulls on the second person. Kiesler $(1983,1996)$ describes a series of principles of interpersonal complementarity. Of primary importance are the principles of correspondence on the affiliation dimension and reciprocity on the control dimension. The principle of correspondence states that interpersonal behaviors that are friendly invite friendly responses and behaviors that are hostile invite hostile responses. The principle of reciprocity states that behaviors that are dominant invite submissive responses and behaviors that are submissive invite dominant responses. Additionally, 
interpersonal principles state that more extreme interpersonal behaviors exert a stronger interpersonal pull on the other to complement this behavior. Kiesler (1983) states, Interpersonal complementarity and noncomplementarity operate precisely only within the same level or intensity of behavior. That is, interpersonal actions at a particular level of intensity tend (with a probability significantly greater than chance) to initiate, invite, or evoke from interactants complementary responses at the equivalent level of intensity (mild-moderate actions pull mild-moderate complementary responses, extreme acts pull extreme responses). (p. 203)

Also, people who more rigidly adhere to a particular pattern are less likely to deviate from this pattern, even when pulled to do so. Kiesler (1983) states, The more extreme and rigid (maladjusted) the interpersonal style of Interactant B, the less likely he or she is to show the predicted complementary response to the interpersonal actions of Person A. An important exception occurs when the predicted complementary response to A falls at the exact segments that define B's extreme and rigid style. (p. 206)

A great deal of research has supported these principles of complementarity. For example, Tracey (1994) found strong support for the principles of complementarity when controlling for the base rate of the interpersonal behaviors when rating the interactions of female dyads. Another example is the Horowitz et al. (1991) study, which found support for the principle of reciprocity. The authors found that depressed people with submissive styles tended to elicit dominant reactions. 
Tracey (1993) made the point that the principles of complementarity described above are probabilistic, not deterministic. Complementarity should be understood in terms of pulls to engage in a certain pattern of behavior that may or may not correspond with how a person typically behaves in interpersonal situations. People with more rigid and extreme interpersonal patterns will more readily resist pulls for behavior that deviates from their typical interpersonal style (e.g., dominant behavior from a typically submissive person). When a person rigidly adheres to an extreme set of interpersonal behaviors it is anxiety provoking for them to deviate from their typical interpersonal style.

Additionally, people with more rigid and extreme patterns are more likely to pull others to enact behaviors that are complementary to their pattern. Kiesler (1996) states, "The maladjusted individual, thus, is an interactant who rarely exhibits the complementary behavior to anyone - except in the relatively rare instances during which her or she is interacting with a perfectly complementary partner” (p. 106). Engaging in interpersonal behaviors that are noncomplementary to a person's typical pattern of interacting is anxiety provoking because it involves deviating from a pattern of behaving that is comfortable and safe. While noncomplementarity can contribute to problematic relations, complementary interactions can improve outcomes of interactions. For example, Estroff and Nowicki (1992) found that complementary dyads performed better than noncomplementary dyads on cooperative tasks (e.g., jigsaw puzzles). This result was true whether their complementarity was hostile or friendly, i.e., a hostile-dominant person with a hostile-submissive person or a friendly-dominant person with a friendlysubmissive person. Thus, complementarity can have important effects on outcomes of a number of different types of interactions. 


\section{Interpersonal Complementarity and Psychotherapy Outcomes}

A great deal of research has examined the role of interpersonal complementarity in psychotherapy outcomes. In his review of the complementarity literature, Kiesler (1996) found a consistent pattern across studies of individual therapy (e.g., Tracey, 1987), as well as family therapy (e.g., Laird \& Vande Kemp, 1987). He states, In the case of successful psychotherapy, the patient and therapist will move from rigid and extreme complementary transaction early in therapy, to non-complementary positions in the change-oriented middle phases of therapy, to a later transactional pattern that exhibits mild and flexible complementarity. (261)

A series of studies by Tracey and colleagues has strongly and consistently supported this model (e.g., Tracey, 1987, 1993; Tracey \& Ray, 1984; Tracey, Sherry, \& Albright, 1999). Tracey et al. studied a sample of 20 clients with successful outcomes treated with cognitive-behavioral therapy. Using growth curve analyses, the authors found that successful clients showed a pattern where in the first stage, therapist and client have relatively high complementarity, in the middle stage low complementarity, and in the end stage, increasing complementarity accompanied by resolution of interpersonal conflict. This U-shaped pattern of complementarity in cases with successful outcomes suggests that initial complementarity and later noncomplementarity may be important to outcomes. Complementarity in the beginning stage of therapy may lead to a sense of security for the client in the therapeutic relationship, as it allows the client to engage in interpersonal behaviors that are consistent and comfortable (Kiesler, 1996). 
Research has supported that early complementarity is an important predictor of positive process and outcome in psychotherapy. Shim and Chung (1998) found that early complementarity predicted the strength of the working alliance. Additionally, the authors found that in successful psychotherapy cases, early complementarity tended to be higher than in unsuccessful cases. Kiesler and Watkins (1989) found that early complementarity was associated with client and therapist ratings of the alliance. In his study of psychotherapy clients, Tracey (1986) found that those clients who did not have high levels of complementarity with their therapists early in therapy tended to terminate prematurely. Caspar, Grossmann, Unmussig, and Schramm (2005) found that higher complementarity predicted better client-rated outcomes. The current study examined the role of early complementarity as a predictor of positive psychotherapy outcomes.

Research has supported that noncomplementarity in the middle phase of therapy is also related with success in therapy. Dietzel and Abeles (1975) hypothesized that successful outcomes of psychotherapy are dependent upon the timing of complementarity levels at the different phases of psychotherapy. Observers rated 20 client and therapist dyads on the Interpersonal Checklist (ICL) in the early, middle, and late phases. They found no differences between successful and unsuccessful cases in the early phase, lower level of complementarity for successful cases in the middle, and no difference in the later phase. However, successful cases had significantly higher complementarity in the end phase than in the middle phase. Henry, Schacht, and Strupp (1986) found a significant relationship between noncomplementary therapist responses and positive therapy outcomes. Brokaw (1983) also found a relationship between noncomplementarity of therapist response and client change, as well as complementary therapist response and 
stable client behavior, i.e. continuation of maladaptive interpersonal patterns. Taken together, these findings suggest that noncomplementary response particularly in the middle phase of therapy is associated with positive outcomes.

Previous research has shown that therapists may not naturally tend to provide noncomplementary response in the middle phase of therapy. Coulter (1993) had beginning therapists, interns, and experienced therapists respond to 16 standardized statements (i.e., representing the range of interpersonal behaviors) from a client who they were told was in the middle stage of therapy. Therapists of all experience levels tended to respond to client statements with friendly-dominant behaviors regardless of the interpersonal stance of the client's statement. There were no differences in the degree of noncomplementarity regardless of experience level. Thus, these therapists consistently responded in a way that encouraged friendly-submissive client behaviors and highly discouraged hostile-dominant client behaviors. Celani (1974) also found that therapists largely tended to behave in friendly-dominant ways in psychotherapy. Thus, these therapists consistently responded in a way that would encourage friendly-submissive client behaviors and highly discouraged hostile-dominant client behaviors. Muran, Segal, Samstag, and Crawford (1994) found that client report of friendly-submissive interpersonal problems were positively associated with early therapeutic alliance, while hostile-dominant problems were negatively associated with early alliance. One explanation for this finding may be that therapists who tend to be friendly-dominant will have a high degree of complementarity with friendly-submissive clients and a high degree of non-complementarity with hostile-dominant clients. Overall, this research 
suggests that therapists may be inclined to take a friendly-dominant stance with clients across stages.

An important component of TLIPT is that therapists shift from more complementarity to less complementarity from the beginning to the middle stage of therapy. Therapists utilize a conceptualization meeting in TLIPT to develop tools to disengage from complementary interaction as the dyad enters the middle phase of therapy. These tools include interpersonal targets (e.g., client needs to be more friendlydominant, therapist needs to be more friendly-submissive). These targets are designed to allow the therapist to disengage from complementary interaction and to disengage from confirming the maladaptive interpersonal pattern after the dyad enters the middle phase of therapy. This study examined if complementarity between TLIPT therapists and clients had significant reductions from the beginning stage of therapy to the middle stage.

Research has suggested that successful therapy outcomes may be associated with a pattern of complementarity following a high-low-high curve, where complementarity in the beginning stage is at a high point, followed by low complementarity at the middle phase of therapy, and high complementarity at the end phase of therapy. However, research has also suggested that therapists may not be inclined to shift complementarity. This study examined the role of complementarity on the affiliation dimension and the control dimension in the beginning phase and middle phase of therapy in bringing about interpersonal change. Additionally, this study explores if TLIPT dyads' complementarity on the affiliation and control dimensions tend to reduce from the beginning to the middle phase of therapy.

Affiliation Complementarity 
Studies of complementarity have highlighted the importance of affiliation complementarity, that is therapist and client matching on being hostile or friendly. In addition to the research described above, several studies have found an association between matching on the friendly side of the affiliation dimension and positive psychotherapy outcomes. Tasca and colleagues (1988; Tasca \& McMullen, 1993) studied one case with successful outcomes and one case with unsuccessful outcomes for each of four therapists in Strupp’s (1980a, 1980b, 1980c) Vanderbilt I research project. The authors coded within session interactions with the Structural Analysis of Social Behavior (SASB, Benjamin, Giat, \& Estroff, 1981) and found that successful cases had a pattern of high complementarity in the beginning stage, then lower complementarity in the middle stage, and finally high complementarity at the end of therapy. In the unsuccessful cases, the degree of complementarity remained relatively stable. Thus, the successful cases in this study tended to match the high-low-high pattern described earlier, while the unsuccessful cases did not. Further, most of the complementarity in the successful cases was in the friendly range of interpersonal behaviors. In the unsuccessful cases, there was a higher degree of non-complementary exchanges throughout therapy than in successful dyads, particularly along the affiliation dimension. Thus, matching on affiliation appeared to be an important factor for differentiating those with and without successful outcomes. Henry et al. (1986) and Svartberg and Stiles (1992) found that corresponding with friendly complementarity along the affiliation dimension was associated with positive therapeutic processes and outcomes, while noncomplementary communications disrupted positive process and outcome. Rudy, McLemore, and Gorsuch (1985) found that complementarity on the affiliation dimension was associated 
with therapist and client rating therapy as successful, however affiliation complementarity was not a strong predictor of other outcomes including symptom reduction and therapist ratings of the client change. Weinstock-Savoy (1986) found that affiliation complementarity was associated with outcomes as rated by observers, therapists, and clients. Thus, complementarity along the affiliation dimension, and particularly friendly complementarity, may be an important contributor to outcome.

While the above described research has suggested that frequent affiliation complementarity, particularly friendly complementarity, is associated with better outcomes, other research suggests that complementarity in hostile interactions also carries weight in determining psychotherapy process and subsequent outcomes. Kiesler and Watkins (1989) examined the relationship between therapist and client complementarity and therapeutic alliance in the third session of therapy. The authors found that higher hostile complementarity was significantly related to higher levels of client rated alliance and that higher levels of overall complementarity were significantly related to higher levels of therapist rated alliance. The authors observed that hostile behaviors were much less prominent in the dyads than friendly behaviors. The authors suggest that these results indicate that while hostile complementarity may be more infrequent in successful therapy dyads, it plays an important role in affiliation complementarity and the development of the working alliance as perceived by the therapist or patient. Wagner (1995) suggests that hostile complementarity may lead to high alliance when hostility is the usual, baseline, comfortable behavior of the client, but will not when hostility is unusual or uncomfortable for the client. Thus, it may be that hostile complementarity is more relevant for the development of a secure therapeutic 
environment early in therapy when hostility is part of the client's typical interpersonal style. Outside of the therapeutic relationship, Campbell and Brown (1990) found that among married couples marital satisfaction was more strongly associated with complementarity in hostile interactions than in friendly interactions. So while research has supported the importance of friendly complementarity on the affiliation dimension, overall affiliation complementarity including hostile complementarity appears to play an important role in the development of therapeutic alliance, an overall secure therapy environment, and perhaps ultimate therapy outcomes. Thus, overall it appears that complementarity along the affiliation dimension, in both friendly and hostile interactions, may be an important contributor to psychotherapy process and outcomes.

\section{Control Complementarity}

Little research has examined the impact of control complementarity on psychotherapy outcomes. Unpublished research by Muran and colleagues (Muran, Samstag, Jilton, Batchelder, \& Winston, 1992a, 1992b) suggests that complementarity on the control dimension may be important to outcome. Muran (1993) indicated that a moderate amount of therapist dominance with a reciprocally moderately submissive client may lead to the best outcomes. Raymond, Friedlander, Heatherington, Ellis, and Sargent (1993) discussed a case study of family therapy. The authors found evidence of a pattern of high-low-high control complementarity over the course of therapy and that this pattern of complementarity may have been linked with changes in the family's rigid interpersonal style. This study provided further empirical examination of the impact of control complementarity on psychotherapy outcomes. 
Research has suggested that complementarity in successful cases follows a pattern of being at a high level at the beginning stage of therapy, lower at the middle stage of therapy, and at a high level at the end stage of therapy. Further, research has shown that complementarity along the full spectrum of the affiliation dimension, i.e., friendly to hostile, and of the control dimension, i.e., dominant to submissive, may play an important role in outcome. Although, much of the previous research described has not examined interpersonal change as an outcome, it is expected that interpersonal change will be associated with complementarity in similar ways. Specifically, it was expected that for both control complementarity and affiliation complementarity a high degree of complementarity in the beginning phase of therapy would be associated with larger interpersonal changes. It was also expected that greater noncomplementarity, that is lower complementarity, in the middle phase of therapy would be associated with greater interpersonal changes.

\section{Working Alliance}

Although there may be conceptual similarities between complementarity and alliance, key differences exist among these constructs that distinguish them from one another. Kiesler and Watkins (1989) state,

Interpersonal complementarity addresses a component of the patienttherapist relationship, distinct from the therapeutic alliance, consisting of the degree of fit of the interpersonal transactions between patient and therapist. In contrast to the working alliance that conceptualizes the conscious and realistic relationship, interpersonal complementarity emphasizes the automatic and distorted relationship resulting primarily, 
but not exclusively, from the patient's rigid and extreme parataxic distortions (Sullivan, 1953a,b).(p. 183)

The authors went on to correlate complementarity and working alliance, and found significant but not perfect correlations (i.e., ranging from $r=-0.33$ to -0.43 ), indicating that as complementarity increased (high complementarity on their measures was indicated by negative numbers), working alliance also tended to increase. Thus, while some variance is shared by these constructs, there are limitations to the overlap. Alliance was examined as a separate contributing factor to interpersonal change in TLIPT clients in this study.

A large body of research has examined the role of the therapeutic working alliance on the outcome of psychotherapy. Several meta-analyses have attempted to summarize this body of literature on the strength of the relationship between alliance and outcome. Horvath and Symonds (1991) using meta-analytic techniques, examined 24 studies based on 20 data sets. They found a highly statistically significant average effect size (.26) linking alliance to outcome. They also found that there was no difference in the relationship regardless of the combination of raters for the alliance and outcomes (i.e., homogenous and heterogeneous combinations of client and therapist ratings of alliance and outcomes). Within this meta-analysis, the authors found that type of treatment, sample size, length of treatment, or if the study was published or unpublished did not have significant effects on the alliance-outcome relationship. In a more recent metaanalysis, Martin, Garske, and Davis (2000) examined 79 studies and found an effect size linking alliance to outcome of .22. Horvath and Bedi (2002) analyzed studies published through the year 2000, thus updating Martin et al.’s (2000) analysis, which only included 
publications since 1997. Horvath and Bedi (2002) found that an average effect size of .21 and a median effect size of .25. More recent studies have continued to show a significant relationship between alliance and outcome in process-experiential psychotherapy (Watson \& Gellar, 2005), brief interpersonal therapy (Wettersten, Lichtenber, \& Mallinckrodt, 2005), and CBT for panic disorder (Casey, Oei, \& Newcombe, 2005).

Similar to complementarity, working alliance appears to follow a stage model in successful therapy. Horvath and Luborsky (1993), in their review of literature, their view of a stage model of the therapeutic alliance. The first stage, within the first five sessions, involves building trust and collaboration, agreement on goals, developing confidence in the procedures and structure of therapy, and the client agreeing to continue with the therapist on the path to change. The second stage involves the therapist beginning to challenge the client's maladaptive patterns, which the client may interpret as reduction in support and sympathy. This may lead the client to act according to dysfunctional interpersonal beliefs and subsequently weaken and rupture the therapeutic alliance. In successful cases, the therapist works to repair the alliance while also disconfirming maladaptive beliefs. In their extensive work on resolution of therapeutic ruptures in the alliance, Safran and Muran (2000) indicate that these rupture and repair cycles are one of, if not the most important element for therapeutic change. That is, the therapeutic relationship is used as a tool for intervention, by first establishing a secure base and subsequently working through problems in the relationship. TLIPT espouses this view as well. 
The stage model for the alliance also follows the stage model for complementarity. That is, the principles of interpersonal complementarity may be utilized within the early, alliance-building phase and the later rupture and repair phase. In the early phase, the therapist is engaged in the client's interpersonal pull and the dyad engages in a high degree of complementarity and alliance building. Therapists then utilize the case formulation to begin to disengage from a complementarity pattern with the client, which is associated with ruptures and reductions in the therapeutic alliance. In the middle phase of therapy, the therapist exerts interpersonal pulls on the client by enacting noncomplementary interpersonal behaviors to the client's maladaptive interpersonal patterns. Thus, research on the stages of psychotherapy has emphasized the importance of high early alliance and early complementarity, and later noncomplementarity in determining outcomes.

Many studies have distinguished between alliance in the beginning stage of therapy and alliance later in therapy. In the Penn Psychotherapy Project, Luborsky (1976) identified two types of alliance. Often more concentrated in the beginning of therapy, Type 1 alliance is rooted in the client experiencing the therapist as being helpful and supportive of the client. More present in the later phases of treatment, Type 2 alliance is a sense of working together against obstacles and towards goals of therapy. In their review of the alliance literature, Horvath and Luborsky (1993) also speculated that there are two distinct phases of alliance. The first phase occurs within the first five sessions and involves the development of collaboration and trust, agreement on goals, and the development of faith in the procedures and frame of therapy. In the second phase, the therapist becomes more challenging of the client after this secure base has 
been established. Thus, it may be that while the development of strong working alliance early in therapy is crucial to success, a strong "Type 1" alliance as described by Luborsky (1976) becomes less important in later stages of therapy when other processes such as interpersonal noncomplementarity become more important. Research has consistently supported that higher working alliance measured in the early phases of therapy (i.e., measured through the fifth session or prior) contributes to better outcomes (e.g., Gaston, 1990; Hartley \& Strupp, 1983; Horvath \& Greenberg, 1986, 1994; Horvath \& Luborsky, 1993; Horvath \& Symonds, 1991; Lambert 1992; Saltzman, Luetgert, Rogh, Creaser, \& Howard, 1976). Thus, previous research has emphasized the role of early alliance in outcomes, as opposed to later, "type 2" alliance.

Further analysis by Horvath and Symonds (1991) found different relationships of the alliance at different stages in therapy to outcomes among the 24 studies they metaanalyzed. They found that alliance rated early and late in therapy had nearly identically high relationships with outcome $(\mathrm{ES}=.31$ and $\mathrm{ES}=.30$ respectively), while alliance averaged over the course of therapy had a lower effect size $(\mathrm{ES}=.21)$. The authors indicate that the difference may be accounted for by the emerging importance of variations in the alliance process that occur in the middle phase. Horvath and Luborsky (1993) indicate that the lower effect size of the relationship between alliance and outcome in the middle stage of therapy may be due to rupture-repair cycles in the middle phases of successful therapies. That is, for therapy to be successful, it is necessary for the therapeutic dyad to build a strong relationship early in therapy. In the middle stage of therapy, the emergence of other important therapeutic processes, such as providing noncomplementary responses, may lead to a decrease in the relationship of alliance and 
outcome in this stage. While much of the previous alliance research has focused on the relationship between early alliance and more symptom-based outcomes, this study focused on the contribution of early alliance to interpersonal change. It was expected that higher early alliances would be associated with greater interpersonal change.

\section{Early Symptom Reduction and Psychotherapy Outcomes}

Research has indicated that in addition to alliance and interpersonal complementarity, early symptom reduction is an important contributor to ultimate outcome. For example, Crits-Cristoph, Demorest, and Connolly (1990) found that early symptomatic improvement (i.e., improvement from baseline to sessions two, three, or four) significantly predicted remission of major depressive disorder, generalized anxiety disorder, obsessive-compulsive disorder, and avoidant personality disorder in clients treated with CBT and dynamic psychotherapy. Further, research suggests that working alliance and early symptom reductions each uniquely contribute to ultimate outcomes of psychotherapy. Gaston, Marmar, Gallagher, and Thompson (1991) found that early symptom reduction and working alliance accounted for significant and independent portions of the variance in therapy outcome. Barber, Crits-Cristoph, and Luborsky (1992) found that early alliance and early gain in therapy contributed each uniquely to outcomes of psychodynamic psychotherapy. It appears that early improvements in therapy may contribute to ultimate outcomes, which may be due to factors including instilling hope in the client and confidence in the therapist. It is expected that early symptom gains, similar to early alliance and complementarity, may contribute to the establishment of an early therapeutic environment that is likely to lead to the ultimate outcome of overall interpersonal pattern change. By establishing this environment, 
therapist and client dyads are more enabled to successfully move into the unstable middle phase of therapy.

Research has indicated that symptom reduction and changes in interpersonal patterns can both result as outcomes of therapy, although several studies have indicated that symptom change often precedes interpersonal change. Several studies (DaviesOsterkamp, Strauss, \& Schmitz, 1996; Wilbereg et al., 1998) have indicated that both interpersonal distress reduction (i.e., the amount and degree of interpersonal problems) and interpersonal pattern change occurred as outcomes intensive group therapy. Horowitz et al. (1988) found that general symptom distress as measured by the Symptom Checklist-90-R (SCL-90-R, Derogatis, 1983) and interpersonal problem distress score as measured by the Inventory of Interpersonal Problems (IIP, Horowitz, et al. 1988) both decreased significantly over the first ten sessions of therapy. Measures of symptoms did not decline in the second 10 weeks of therapy, while IIP distress scores decreased significantly. The authors state that symptom distress score may have reached a point where there was little room for further improvement, while interpersonal problems continued to improve. Thus, early symptom reductions may contribute to the establishment of a secure environment in the beginning stage of therapy. Building upon the security, the therapist and client may then engage in the middle stage of therapy in which may ultimately contribute to changes in maladaptive interpersonal patterns.

Although reduction in symptoms can often occur in the beginning stage of therapy, changes in interpersonal pattern may take more time. Kopta, Howard, Lowry, and Beutler (1994) suggest that characterological aspects of clients such as maladaptive interpersonal patterns are more resistant to change than are symptoms. Thus, changes in 
interpersonal patterns may take more time than symptom improvements. This sentiment was echoed in research by Schauenburg, Kuda, Sammet, and Strack (2000). They found that in a sample of clients treated with a mean of 7.8 short-term psychodynamic psychotherapy sessions, IIP interpersonal distress reduced, but no significant changes were found on measures of interpersonal pattern change. The authors suggest the lack of change in interpersonal patterns reflects the trait-like qualities of interpersonal patterns and the lack of treatment duration to change these. In the current study, it was expected that symptom reduction would occur, particularly in the beginning phase of therapy and that this would subsequently help establish an environment in which changes in trait-like maladaptive interpersonal patterns are more likely to occur.

Preliminary research with the TLIPT sample indicated that symptom outcomes follow stage like patterns that seem correspondent to those described in complementarity and alliance research (Salsman, McGuffin, \& Murrell, 2002). The authors utilized linear, quadratic, and cubic regression to examine patterns in outcome measures with overall successful clients over stages of therapy. In addition to significant linear reductions in symptoms, the authors found a cubic pattern where the clients had significant symptom reductions in the early alliance-building stage of therapy, a leveling off of symptom reductions in the middle phase of therapy, and a reduction in symptoms again at the end, resolution stage of therapy. This suggests that early symptom reductions may be an important predictor of overall success in therapy. These early symptom reductions may contribute to the creation of a secure early environment in therapy, which eases the transition to the middle phase of therapy. It is expected that early symptom reductions will be associated with interpersonal changes. 


\section{The Present Study}

This study examined, first of all, if interpersonal changes along with symptom reductions occurred in clients treated with TLIPT. Much research has focused on symptom-based outcomes of therapy, however less research has examined changes in interpersonal patterns. This study examined how therapy focused on providing positive outcomes through interpersonal changes (i.e., TLIPT), actually affects interpersonal change. This study particularly examined the areas of interpersonal affiliation, control, and rigidity and extremity.

Research supports the effectiveness of approaches similar to the TLIPT approach for the treatment of a variety of problems (Anderson \& Lambert, 1995; Crits-Christoph, 1992; Elkin et al., 1989; Holm-Hadulla, 1995; Shapiro et al., 1994). A preliminary evaluation of TLIPT by Murrell, Mehl, Buck, Nichols, and Fearing (1999) found that clients improved on measures of symptoms. Although research supports the efficacy of this therapy in reducing symptoms, theory points to the importance of a change in the core pathology that causes the problems, in this case maladaptive interpersonal patterns, in order for symptom reductions to be maintained (Muran et al., 1995; Rosenthal et al., 1999). This study examined if interpersonal changes do occur in clients treated with TLIPT.

The second part of this study took an exploratory approach to how the interpersonal changes that occur in TLIPT clients come about. Several variables have shown promise as predictors of more symptom-based outcomes and show potential as predictors of interpersonal change. These predictors include variables that are thought to contribute to a secure therapeutic environment in the early phase of therapy, including the 
working alliance, early therapist and client complementarity on the affiliation and control dimension, and early symptom reduction. Wampold (2001), in his discussion of the factors common to all forms of psychotherapy, highlights the primary importance of a healing context leading to therapeutic change. This healing context includes elements of a secure early therapeutic alliance, including the development of the relationship (e.g., alliance and complementarity). TLIPT therapists conduct therapy in a manner which encourages building alliance, engaging in high early complementarity, and subsequent symptom reductions in the beginning phase of therapy. This study explored if these elements contribute to subsequent interpersonal change. Further, theory and research indicate that less complementarity in the middle phase of therapy may also impact overall interpersonal change. TLIPT also encourages disengaging from maladaptive patterns in the middle phase of therapy, i.e., increasing noncomplementarity. The TLIPT approach conceptualizes therapy as a relationship built to modify idiographic maladaptive patterns through complementary and noncomplementary interactions. This study explored if noncomplementarity in the middle phase of therapy contributes to subsequent interpersonal change. In order to explore the variables hypothesized to contribute to interpersonal change, this study compared those with high interpersonal changes with those without high interpersonal changes on these variables.

A case study of a TLIPT client (Shields, Murrell, \& Salsman, in press), illustrates what may be the model of change for clients treated with this therapy with successful outcomes. Data from the client's case support that symptom reductions, reductions of interpersonal problems, and interpersonal pattern changes occurred in this client treated with TLIPT over 45 sessions and were maintained through measurement at a one-year 
follow-up. In the early stage of therapy, the authors found significant increases in the therapeutic alliance, significant reductions in symptoms, and significant reductions in interpersonal problems and distress. In subsequent stages of therapy, the authors found significant changes in the client's interpersonal patterns. Specifically, the authors found that the client increased her friendliness and decreased hostility on the affiliation dimension, as well as increased dominance and decreased submissiveness on the control dimension. Interpersonal patterns measured at the end of therapy also revealed reductions of rigidity and extremity and interpersonal pattern across several different measures. The authors suggest that these changes occurring over 45 sessions are consistent with findings of Howard, Kopta, Krause, and Orlinsky (1986) showing that a greater number of sessions is associated with greater improvement. The authors also indicate that the interpersonal changes appeared to be related to the therapist's ability to respond in non-complementary ways after building a secure therapeutic environment, which includes ingredients such as a strong alliance, interpersonal complementarity, and early symptom reductions. This study expanded on these findings by studying interpersonal change with a sample of TLIPT clients and with a variety of measures of interpersonal patterns.

Connolly Gibbons (2004) asserts that psychotherapy research should examine multiple outcomes including decreased symptoms, improved interpersonal functioning, and improved self-understanding of maladaptive interpersonal patterns. The process of improving self-understanding involves learning new and adaptive interpersonal behaviors in the context of the therapeutic relationship. TLIPT, based on similar principles, works to achieve decreased symptoms through utilization of the therapeutic relationship, 
improved interpersonal understanding, and increased adaptive interpersonal behaviors. This study approached the assessment of change in symptoms and underlying interpersonal patterns based on a multi-measure, multi-rater approach. This study measured decreases in symptoms through self-report measures of symptoms; improved understanding of maladaptive interpersonal behaviors through changes on self-report interpersonal circumplex measures (e.g., IIP); and increases in adaptive interpersonal behaviors through interpersonal measures completed by the client, therapist, observer, and clients’ significant others.

\section{Hypotheses}

(1) TLIPT clients will have decreased:
a. symptoms
b. interpersonal distress
c. interpersonal rigidity and extremity

(2) TLIPT clients will have increased:

a. friendliness on the affiliation dimension (i.e., reduced hostility)

b. dominance on the control dimension (i.e., reduced submissiveness)

(3) From the beginning stage of therapy to the middle stage of therapy, TLIPT client and therapist dyads will have significant reductions in:
a. control complementarity
b. affiliation complementarity

(4) TLIPT clients with larger overall reductions in rigidity and extremity of their interpersonal patterns will have significantly:

a. higher early alliance 
b. higher early symptom reduction

c. higher early complementarity on the affiliation dimension

d. higher early complementarity on the control dimension

e. lower middle stage complementarity on the affiliation dimension

f. lower middle stage complementarity on the control dimension than those with stable or increasing patterns of rigidity and extremity.

(5) TLIPT clients with larger overall increases in friendliness on the affiliation dimension will have significantly:

a. higher early alliance

b. higher early symptom reduction

c. higher early complementarity on the affiliation dimension

d. higher early complementarity on the control dimension

e. lower middle stage complementarity on the affiliation dimension

f. lower middle stage complementarity on the control dimension than those with stable or decreasing friendliness on the affiliation dimension.

(6) TLIPT clients with larger overall increases in dominance on the control dimension will have significantly:

a. higher early alliance

b. higher early symptom reduction

c. higher early complementarity on the affiliation dimension

d. higher early complementarity on the control dimension

e. lower middle stage complementarity on the affiliation dimension

f. lower middle stage complementarity on the control dimension 
than those with stable or decreasing dominance on the control dimension. 


\section{METHODS}

This project is part of an ongoing study examining TLIPT among a sample of clients seen at a graduate student training clinic at a large urban university. Various measures were completed by clients, therapists, and observers based on a predetermined data collection schedule, which was developed prior to the design of this study.

\section{Participants}

\section{Clients}

Clients were volunteers who consented to participate in an ongoing project that examines the process and outcome of TLIPT. Most of the referrals for this clinic were seen for reduced fees (i.e., on a sliding fee scale) and came from the community rather than from university sources (i.e., clients were typically not students, faculty, or staff of the university). After an initial phone screening, therapy clients participated in a semistructured intake interview and a battery of intake questionnaires. Following the intake, interviewers and the clinic supervisor determined whether the clinic was an appropriate treatment setting for the client and discussed the most appropriate treatment team among several options. Clients presenting with clearly defined anxiety disorders or psychotic symptoms were seen by therapists in different agency projects. Clients were referred to the TLIPT treatment team within the clinic when they described some form of current interpersonal difficulties (e.g., relationship problems, loneliness, withdrawal, etc.) as a main presenting problem. Other than these general guidelines, inclusion criteria for clients in the study were relatively broad.

After being referred to the TLIPT treatment team, clients were approached to participate in the study. Clients who agreed to participate were assigned a code number 
to protect their confidentiality. Consenting clients were asked to complete questionnaires after every session of a 21-session therapy package and according to the predetermined TLIPT data collection schedule. While clients were initially asked to participate in 21 sessions, many completed fewer or more than 21 sessions. Clients who opted to continue therapy after 21 sessions, began the data collection schedule again after session 21. In order to be included in any of the analyses for this study, clients were required to have data on at least nine sessions. Nine sessions was chosen as a cut point for several reasons. First, research has indicated that interpersonal patterns are resistant to change in therapy lasting under eight sessions (i.e., Schauenburg et al., 2000). Secondly, theory and research have indicated that the early stage of successful therapies (i.e., sessions one through four) typically includes the development of a strong working alliance (e.g., Horvath \& Symonds, 1991), high levels of complementarity (e.g., Tracey et al., 1999), and symptom reductions (Crits-Cristoph et al., 1990). Further, theory and research have indicated that noncomplementarity in the middle phase of therapy may contribute to interpersonal change. Nine sessions was chosen to allow sufficient time for an early stage and a middle stage of therapy to take place. Finally, the data collection schedule of the TLIPT project calls for several important measures of interpersonal pattern to be collected around session nine.

These criteria led to a sample of 61 clients with data from nine or more sessions. The 61 clients (23 male and 38 female; 47 Caucasian, 5 African American, 2 Asian American, 3 Hispanic American, 2 other, and 2 declined to state their race) were seen by 28 clinical psychology Ph.D. graduate students at a University-based training clinic. The clients' ages ranged from 19 to 66 years $(M=31.75, S D=10.21)$. Clients in this sample 
had therapy lengths ranging from 9 to 76 sessions $(M=24.85, S D=16.53)$. Twenty three clients had therapy range from 9 to 15 sessions, 14 clients had therapy ranging from 16 to 21 sessions, and 24 clients had therapy length greater than 21 sessions.

\section{Therapists}

All therapists were clinical psychology $\mathrm{Ph}$. D. graduate students who agreed to participate in this project. Therapist training included readings on the foundations and techniques of interpersonal therapy by authors such as Levenson (1995), Safran and Muran (2000) and Teyber (2000); observing videotapes of weekly therapy clients treated with TLIPT; taking a semester long course on the interventions of interpersonal therapy; and discussions of clients on a weekly therapy "team” made up of their peers and supervised by the designer of TLIPT. Therapists were supervised weekly on the therapy "team" and individually on a periodic basis and at any time upon request. Therapists complete measures rating the clients' interpersonal patterns after every session.

Among the 28 total therapists, one therapist saw six clients, three therapists saw four clients, five therapists saw three clients, nine therapists saw two clients, and ten therapists saw one client. Of the 28 total therapists, there were 11 male and 17 female; 25 Caucasian, 2 Hispanic, and 1 Asian-American therapist. There were 20 therapists between the ages of 20 to 30, 4 therapists between the ages of 30 to 35, and 4 therapists greater than age 35.

\section{Measures}

Working Alliance Inventory - Short Form (WAI; Horvath \& Greenberg, 1986, 1989; Tracey \& Kokotovic, 1989). 
The WAI is a 12-item self-report questionnaire designed to measure the strength of the therapeutic relationship. The 12-item version is shortened from the original 36item version after a refinement by Tracey and Kokotovic (1989). Clients rate items such as "I believe (therapist’s name) likes me” and "(therapist's name) and I trust one another" on a seven-point Likert scale ranging from never (1) to always (7). Two items are reverse scored. Scores are calculated by summing the items, after reverse scoring is applied. Total scores range from 12 to 84 .

In a meta-analysis of 79 studies, Martin et al. (2000) found that client ratings on the WAI had a mean internal reliability of .84. A sample of TLIPT clients' first session WAI scores had an alpha of .88. Research also suggests that client ratings, as opposed to therapist or observer ratings, have higher correlations with outcome (Asay \& Lambert, 1999). Clients completed the WAI after every session.

Outcome Questionnaire 45.2 (OQ45; Lambert et al., 1996).

The OQ45 is a 45-item self-report questionnaire designed to measure client progress in therapy on a variety of symptoms from session to session. The scale was designed for the specific purpose of being administered on a repeated basis. The items were designed to measure the degree clients experience the most common symptoms of frequently occurring psychiatric disorders. Clients rate items such as "I feel no interest in things" and "I feel nervous" on a five-point Likert scale ranging from never (0) to almost always (4). Items indicating a positive outcome are reverse scored. The questionnaire yields a total score, which is a measure of overall outcomes; a symptom distress subscale, which specifically examines symptoms of anxiety, mood, adjustment, and stress related disorders; an interpersonal relations subscale, which examines problems with loneliness 
and family, marital, and social conflicts, and a social roles subscale, which examines problems in roles at work, school, and home. The subscale scores are calculated by summing the items which make up the subscale, and the subscale scores are summed to calculate the total score. The total score can range from 0 to 180 . Higher scores on these scales reflect greater symptoms or problems.

The OQ45 has been found to have internal consistency above .90 for total score in both undergraduate and clinical populations, and to have test-retest reliability with an alpha of 0.84 (Lambert et al., 1996). A sample of TLIPT clients had alphas of .96 for first session OQ45 total scores, .96 for the symptom distress subscale, .78 for the interpersonal relations subscale, and .83 for the social roles subscale. The authors also report significant Pearson product-moment correlations between the OQ45 and numerous other measures of outcome including a correlation of .80 with the BDI (Beck, Ward, Mendelson, Mock, \& Erbaugh, 1961), a correlation of .78 with the General Severity Index of Symptom Checklist-90-R (Derogatis, 1983), a correlation of .64 with the Social Adjustment Scale (Weissman \& Bothwell, 1976), and a correlation of .64 with the StateTrait Anxiety Inventory (STAI; Spielberger, Gorsuch, \& Lushene, 1970). Clients completed the OQ45 after each session.

Interpersonal Outcome Measures

Inventory of Interpersonal Problems - Circumplex Version (IIP, Alden et al. 1990; Horowitz et al.,1988). On the IIP, clients were instructed to rate the degree to which they experience a wide range of interpersonal problems. The IIP is a 127-item self-report questionnaire designed to measure the degree of interpersonal problems a person is experiencing. Clients respond to items that begin with one of two stems, i.e., 
"It is hard for me to..." (78 items, e.g., "trust other people") or items with the format "I ... too much" (49 items, e.g., "I argue with other people too much"). The items are rated on a five-point scale ranging from "not at all” (0) to "extremely" (4). In this study the IIP interpersonal distress score, which is the mean of the 127 items and thus has a range from 0 to 4 , was used as a measure of outcome. The IIP distress score represents the degree a client experiences overall problems in interpersonal relationships. The IIP distress score has been shown to have a test-retest reliability of .98 (Horowitz et al., 1988). A sample of TLIPT clients' first session IIP distress scores had an internal reliability of .98. Clients completed the IIP prior to beginning therapy, at the ninth session, and at the eighteenth session.

A subset of 64 items is utilized to calculate several measures of clients' interpersonal patterns. The IIP has been geometrically formatted to be scored as a circumplex (Alden et al., 1990). A person's scores can be utilized to create a picture of the person's interpersonal pattern on a two-dimensional, 360 degree plane. Thus, scores are computed for each client on the control axis, the first dimension running from top to bottom, and on the affiliation axis, the second dimension running from left to right. Negative scores on the control axis (i.e., in the bottom half of the plane) indicate submissiveness, whereas positive scores (i.e., in the top half of the plane) indicate dominance. Negative scores on the affiliation axis (i.e., in the left half of the plane) indicate hostility, whereas positive scores (i.e., in the right half of the plane) indicate friendliness. In order to calculate scores on the control and affiliation axes, scores are calculated on eight interpersonal scales for each of the combinations of control and affiliation behaviors (i.e., hostile-dominant, hostile, hostile-submissive, submissive, 
friendly-submissive, friendly, friendly-dominant, and dominant). Each of the octant scores are calculated by taking the means of eight items corresponding to each octant (e.g., "I am too independent” for problems with dominance, also described as being domineering; "It is hard for me to be supportive of another person's goals in life" for problems with hostile dominance, also described as being vindictive; "I keep other people at a distance too much" for problems with hostility, also described as being cold; "It is hard for me to socialize with other people" for problems with hostile submissiveness, also described as being socially avoidant; "It is hard for me to be assertive with another person" for problems with submissiveness, also described as being nonassertive; "I let other people take advantage of me too much" for problems with friendly submissiveness, also described as being exploitable; “I am affected by another person’s misery too much” for problems with friendliness, also described as being overly nurturing; and "It is hard for me to stay out of other people’s business” for problems friendly dominance, also described as being intrusive). The means are then subjected to an algorithm that transforms them into eight standard scores where the mean is 40 and the standard deviation is 10. The standardized scores thus serve as measures of the amount the person experiences problems with interpersonal behaviors within each of the octants, for example a high score on hostile dominance indicates that the person has a pattern of being vindictive. Horowitz et al. (1988) report internal reliability of the octant scales ranging from .82 to .94 and test-retest reliability ranging from .80 to .98 . Alden et al. report internal reliabilities of the circumplex version octant scales ranging from .72 to .85 and adequate test-retest reliability. A sample of TLIPT clients had internal reliabilities on first session IIP octant scores ranging from .75 to .95. 
Scores for the control and affiliation dimensions are calculated through algorithms using the eight standardized octant scores. Alden et al. (1990) and Horowitz et al. (1988) found strong support for a two dimensional structure underlying the IIP, with the two dimensions corresponding to control and affiliation. Alden et al.’s findings also indicate that the IIP adequately converges with other interpersonal circumplex measures.

Scores on the IIP can also be used to calculate a measure of rigidity and extremity called vector length. Vector length scores are calculated using the control and affiliation scores. Geometrically, the control score represents the vertical axis, while the affiliation score represents the horizontal axis. From the scores on these two dimensions, an IIP summary point indicating a point on the 360 degree plane can be plotted. Conceptually, this summary point indicates the one point in the interpersonal circle that is indicative of the respondent's average interpersonal behavior (e.g., a negative affiliation score and a negative control score would indicate a summary point within the hostile submissive octant). The vector length score is the measure of the distance of the summary point from the center. Conceptually, a vector length of zero would indicate a summary point in the center of the interpersonal circumplex, and thus a perfect balance among all interpersonal behaviors. Larger vector length scores indicate more rigid adherence to a particular interpersonal style. Vector length scores also indicate interpersonal extremity because as the vector length increases, the farther the summary point is from the center of the circle, and the more extreme the corresponding interpersonal behavior associated with the summary point.

Impact Message Inventory - Circumplex Version (IMI, Kiesler \& Schmidt, 1993). The IMI is designed to measure a person's interpersonal pattern from the perspective of 
another individual interacting with that person. In other words, the IMI is designed to measure the interpersonal impact that Person A perceives that Person B has had on him or her following an interaction with Person B. Several formats of the IMI were used in this research, however generally the IMI measures the rater's covert reactions to the interpersonal behavior of the target. This circumplex measure consists of 56 items rated on a four-point scale from not at all (1) to very much so (4). Stems of items are, "When I am with this person, he/she makes me feel...", "When I am with this person, he/she makes me feel that...", and "When I am with this person it appears to me that...”. In a similar fashion to the IIP, scores are computed for interpersonal octant scores, the affiliation axis, the control axis, and vector length.

Internal reliability of the octants ranges from .69 to .90 (Schmidt, Wagner, \& Kiesler, 1999). A sample of TLIPT clients had internal reliabilities on first session IMI octant scores ranging from .65 to .86. Schmidt et al. also found strong support for a two dimensional structure underlying the IMI. The two dimensions appeared to correspond with affiliation and control. The authors also found that the IMI appeared to have good circumplex properties.

Therapist ratings of the client, client ratings of the therapist, observer ratings of the client, based on observations of taped sessions, observer ratings on the therapist, based on observations of taped sessions, and clients' significant other ratings of the client were utilized in this project. Therapists rated clients on the IMI after every session, while observers rated clients, observers rated therapists, clients rated therapists, clients' significant others rated the clients on a less frequent but predetermined schedule. Therapist, client and observer ratings were completed directly after sessions or after 
viewing sessions. Significant other ratings were sent home with clients on three occasions (after sessions 1, 9, and 18). The clients subsequently had a significant other complete the IMI and seal it in an envelope, which was returned to the research staff. Impact Message Inventory - Generalized Other Form - Circumplex Version (IMIGO, Kiesler \& Schmidt, 1993). The IMI-GO is a variation of the IMI designed to measure a person's perception of how others have typically interacted with him or her over time. In other words, individuals rate their typical interpersonal approach with others. TLIPT clients and therapists rated their own interpersonal pattern using this selfreport form. This measure is still in circumplex form and consists of the same 56 items rated on a four-point scale from not at all (1) to very much so (4) as the IMI. Although the same items are used for the IMI-GO, stems are stated differently including, "When people are with me, they typically feel...", "When people are with me, they typically feel that they...", and "When people are with me, it typically appears to them that I...”.

As described above, the IMI generally has demonstrated good validity (Strong et al., 1988) and reliability (Schmidt et al., 1999). Little research has been done examining the generalized other format of the IMI, and no research has examined if the IMI-GO is more of a measure of interpersonal traits or transient interpersonal interaction style. As the IMI-GO focuses more on typical patterns of interactions, it may be that the IMI-GO measures more stable, trait-like characteristics of a person’s interpersonal pattern. Support for this hypothesis was provided within this sample by stronger correlations with more stable, trait-like measures of interpersonal patterns (e.g., IIP), than state dependent measures of interpersonal patterns (e.g., IMI). Analyses with session one ratings in this sample indicated that the affiliation score on the IMI-GO had a higher correlation with 
the IIP ( $r=0.39)$, than with the IMI ratings of the client by therapists $(r=0.32$ ), observers $(r=0.28)$, and significant others $(r=0.25)$; and that the control score on the IMI-GO had a higher correlation with the IIP ( $r=0.57)$, than with the IMI ratings of the client by therapists $(r=0.44)$, observers $(r=0.37)$, and significant others $(r=0.44)$. Table 1 summarizes the measures used in this study.

\section{Procedure}

\section{Treatment}

Therapists were trained in Time Limited Interpersonal Therapy (TLIPT, Murrell, 2003) that is based on the theoretical work of Sullivan (1953a) and closely resembles the Brief Relational Therapy approach described by Safran and Muran (2000). Emphases in this interpersonal approach include a collaborative relationship between therapist and client and explicitly addressing and utilizing ruptures in this collaborative relationship (Shields et al., in press). TLIPT is based in interpersonal theory and has tailored its interventions to bring about interpersonal change as the ultimate goal of therapy. The approach draws from the work of Strupp \& Binder (1984), Levenson (1995), Kiesler (1996), Safran and Muran (2000) and Teyber (2000). TLIPT conceptualizes three stages of therapy.

In the beginning stage of therapy the goal is to establish a secure therapeutic environment, which can later be utilized to challenge clients’ maladaptive interpersonal patterns. As with all other interactions, the interactions in therapy are subject to interpersonal patterns, pulls, and complementarity. In fact, TLIPT therapists utilize these elements within the therapeutic relationship as a crucial tool of therapy. Kiesler (1996) 
Table 1

$\underline{\text { Summary of Study Questionnaires }}$

\begin{tabular}{|c|c|c|c|}
\hline Questionnaire & Purpose & Rater & Target of the Rating \\
\hline $\begin{array}{l}\text { Working Alliance Inventory } \\
\text { Short Form (WAI) }\end{array}$ & Measure of the therapeutic alliance & Client & Therapeutic Dyad \\
\hline $\begin{array}{l}\text { Outcome Questionnaire } 45.2 \\
\text { (OQ45) }\end{array}$ & $\begin{array}{l}\text { Measures outcomes including symptom distress, } \\
\text { interpersonal relations, and social roles }\end{array}$ & Client & Client \\
\hline $\begin{array}{l}\text { Inventory of Interpersonal } \\
\text { Problems - Circumplex } \\
\text { Version (IIP) }\end{array}$ & $\begin{array}{l}\text { Measure of general interpersonal style with indicators of } \\
\text { interpersonal rigidity and extremity, affiliation, and } \\
\text { control; also measures interpersonal distress }\end{array}$ & Client & Client \\
\hline $\begin{array}{l}\text { Impact Message Inventory (IMI): } \\
\text { Therapist }\end{array}$ & $\begin{array}{l}\text { Measure of interpersonal impact Person A has on Person B: } \\
\text { - The therapist's impression of the client's } \\
\text { interpersonal approach in therapy sessions }\end{array}$ & Therapist & Client \\
\hline Observer & $\begin{array}{l}\text { - The observer's impression of the client's } \\
\text { interpersonal approach in therapy sessions } \\
\text { based on observations of videotapes }\end{array}$ & Observer & Client \\
\hline Significant Other & $\begin{array}{l}\text { - A significant other's impression of the client's } \\
\text { interpersonal approach in interaction(s) outside } \\
\text { of therapy }\end{array}$ & $\begin{array}{l}\text { Significant } \\
\text { Other }\end{array}$ & Client \\
\hline Client & $\begin{array}{l}\text { - The client's impression of the therapist's } \\
\text { interpersonal approach in therapy sessions }\end{array}$ & Client & Therapist \\
\hline $\begin{array}{l}\text { Impact Message Inventory - } \\
\text { Generalized Other Form } \\
\text { (IMI-GO): }\end{array}$ & $\begin{array}{l}\text { Measure of a person's typical interpersonal pattern } \\
\text { based on his or her perception of the impacts he or she } \\
\text { typically has on others: }\end{array}$ & & \\
\hline Client & $\begin{array}{l}\text { - The client's impression of his or her typical } \\
\text { interpersonal approach with others. }\end{array}$ & Client & Client \\
\hline Therapist & $\begin{array}{l}\text { - The therapist's impression of his or her typical } \\
\text { Interpersonal approach with others. }\end{array}$ & Therapist & Therapist \\
\hline
\end{tabular}


noted that in the beginning stage of therapy, therapists are inevitably hooked into an interpersonal pattern that is complementary with the client's. He states,

Through statements and nonverbal behaviors, the patient sends evoking messages that shape the therapist to respond from a narrow portion of his or her inner experience and behavioral repertoire. The therapist inevitably is pulled to provide the complementary response because the patient is more adept, more expert in this distinctive, rigid, and extreme game of interpersonal encounter. (p. 246)

Kiesler (1996) goes on to state,

The therapist cannot not be hooked temporarily into providing the complementary response to the patient. Indeed, getting hooked eventuates in positive consequences. Becoming hooked probably is necessary for establishment of a working therapeutic alliance; it also permits the therapist to experience firsthand the aversive interpersonal consequences of the patient's maladaptive transactions. (p. 246)

The therapist being complementary in the early phase of therapy subsequently likely contributes to the development of important early stage variables including the therapeutic alliance and early symptom reduction. Thus, through the early stage of TLIPT the therapist focuses on building a strong therapeutic alliance. At the same time, the therapist collaborates with the client to develop an understanding of the client's maladaptive interpersonal pattern and how this pattern may be causing problems. In order to transition from the early stage to the middle stage of therapy, the therapist utilizes his or her own reactions to help understand how the client is pulling the 
therapist to behave interpersonally. Following the beginning stage of therapy, the therapist's task is to begin to engage the client in ways that do not confirm the client's maladaptive interpersonal pattern. As a guide, TLIPT therapists, in conjunction with the supervisor and observer, develop a conceptualization of the client called the cyclical maladaptive pattern (CMP, Levenson, 1995; Schacht, Binder, \& Strupp, 1984) after the fourth session. The CMP helps the therapist to understand the client's models of self and other, the interpersonal pulls the client exerts on others, including the therapist, and the behaviors that are non-complementary to the client's maladaptive pattern. This understanding can be aided by circumplex measures that examine the impact the client is having on the therapist (Wagner, Kiesler, \& Schmidt, 1995). Circumplex measures are used in the CMP to determine interpersonal behavior targets (e.g., friendly-submissive). Therapists employ the CMP to inform their interventions to begin to disengage from a complementary pattern and pull the client to enact new interpersonal behaviors. Thus, the development of the CMP is a marker for transition from the beginning phase to the middle phase of therapy. Client tolerance for non-complementary interactions is theorized to be linked to the security the client and therapist have built through their relationship in the beginning stage of therapy (Kiesler, 1996). Therefore, it is theorized that higher degrees of important therapy variables (e.g., alliance, complementarity, and symptom reductions) in the beginning stage will contribute to greater interpersonal change.

The therapist utilizes several therapeutic tools to disengage from and disconfirm the client's maladaptive interpersonal pattern. For example, the therapist utilizes processing of the client's emotional experiences within the therapeutic relationship as an 
in-vivo learning experience. In processing, clients and therapists discuss their emotional reactions that arise during therapy. Additionally, the dyads communicate about the communication between them, or metacommunicate. Metacommmunication can be an atypical process outside of therapy. However, this process provides clients and therapists opportunities to openly and explicitly discuss and analyze interpersonal behavior. From this negotiation of interpersonal behavior, clients have the opportunity to try out new behaviors.

In the middle stage of TLIPT, the therapists work to provide responses to the client that are non-complementary and thus different than in the past and therefore do not confirm the maladaptive pattern. In their review of negative treatment effects in psychotherapy, Nolan, Strassle, Roback, and Binder (2004) found strong evidence that therapists have difficulty realizing when there are hostile interactions or therapeutic ruptures occurring within the therapy relationship. If these ruptures go unresolved, research indicates that this may lead to negative outcomes. The authors suggest that it is important then for therapists to develop a strong alliance early in therapy and to utilize tools such as complementarity to resolve ruptures. Through repeated trials of noncomplementary interactions TLIPT therapists exert interpersonal pulls on clients to enact behaviors that are different from typical interactions. While these can be uncomfortable interactions and may lead to ruptures in the therapeutic relationship, non-complementary interactions are theorized to be important for bringing about interpersonal change. As suggested by Nolan et al., TLIPT therapists work to engage in non-complementary interactions within the context of a secure therapeutic environment built in the beginning stage of therapy (i.e., one that includes early complementary interactions, a strong 
working alliance, and early symptom reductions). Therapists utilize the CMP conceptualization to inform them of the tact, timing, and dosage of non-complementarity based on the level of security built in the early therapeutic alliance. In addition, therapists utilize metacommunication to resolve any rupture that occurs in the therapeutic alliance. Therapeutic dyads utilize rupture resolution processes as a tool for enacting interpersonal changes (Safran \& Muran, 2000).

In the final stage of therapy, the therapist and client resolve the interpersonal instability of the middle stage, as the therapist and client engage in increasing levels of complementary interactions. This resolution in the final stage comes from therapist and client engaging in complementarity utilizing the new set of interpersonal behaviors negotiated in the middle phase of therapy. Thus, this final stage of therapy reinforces the new interpersonal pattern through the comfort provided through complementarity.

\section{Measurement of Therapist Adherence}

As a measure of therapeutic adherence, therapists and observers rated the degree therapists utilized interventions prescribed by TLIPT and those proscribed by TLIPT after every session. Therapeutic interventions prescribed by the TLIPT approach include responding empathically, exploring affect, focusing internally, addressing therapeutic rupture, processing and metacommunicating about the therapist-client relationship, and exploring client self-concept. Therapeutic interventions proscribed by the TLIPT approach include asking about others, giving advice, focusing on problem-solving, providing information, and challenging cognitions. Although there were distinctions between prescribed and proscribed interventions, therapists were encouraged to apply TLIPT interventions "flexibly" with their clients. This flexibility included encouraging 
therapists to utilize the interventions that best apply within the context of the conceptualization, particularly using the principles of interpersonal complementarity (Kiesler, 1996); the therapeutic alliance; and the stage of therapy, rather than strictly adhering to only interventions prescribed by TLIPT. For example, although giving advice is not prescribed by TLIPT, it would be reasonable for a therapist, after a strong alliance has developed, to nurturingly offer advice (i.e., an interpersonally friendlydominant action) to a client whose typical interpersonal style may be to reject others in a hostile way (i.e., hostile-dominant actions). Thus, this would work toward a goal of interpersonally pulling the client and providing the client opportunities to learn to react in less hostile-dominant and more friendly-submissive ways. Preliminary research on data from the TLIPT sample indicates that a flexible approach, using a small proportion of proscribed interventions is associated with better outcomes than those who rigidly adhere to prescribed interventions or those who have little adherence and use a large proportion of proscribed interventions (Salsman, Ulmer, \& Murrell, 2002).

Analysis Interpretation Strategy

A number of repeated measures ANOVAs were planned for study question one to examine change among the various outcome measures over the course of therapy and a number of one-way ANOVAs were planned for study question two to examine differences in process variables among those with high versus low change. It was decided that an ANOVA approach, as opposed to a MANOVA approach, would be applied. The univariate approach had the advantage of examining each of the measures by different raters individually. That is, this approach avoided losing power to detect changes in individual measures that might occur through conglomerating analyses of the 
measures. For example, this study sought to examine if therapists rated interpersonal change differently than significant others, rather than examining if interpersonal change occurred on average across raters.

Since multiple comparisons were planned, an analysis strategy to avoid finding false positives, or type I errors, was implemented. One strategy to reduce the potential for type I errors would have been to reduce the number of comparisons tested by combining measures, for instance, combining the five measures of clients' interpersonal affiliation (i.e., therapist rating client IMI, observer rating client IMI, significant other rating client IMI, client self-report IMI-GO, and client self-report IIP). However, this strategy was not chosen because an important part of this study was to examine if there were differences in interpersonal changes measured in clients from multiple raters and multiple measures. Instead, the Bonferroni statistical correction was applied. Groups of analyses (e.g., changes in symptom measures, changes in measures of vector length, etc.) were subjected to the Bonferroni correction by dividing the selected alpha level of .05 by the number of analyses in that group. For instance, the five analyses of vector length had to reach an alpha level of .05 divided by five (i.e., the total number of vector length analyses), or .01 in order to reach statistical significance. Therefore, those analyses indicating differences at the Bonferroni corrected level were considered strong evidence of actual change. In spite of the strengths of the Bonferroni correction in controlling type I error, this correction also increases the potential for finding false negatives, or type II errors.

In order to avoid type II errors, a second analysis interpretation strategy was utilized. This strategy involved considering those analyses with an alpha level that did 
not meet the Bonferroni corrected alpha level but was less than .05 to be indicative of a potential trend. This strategy was applied for several reasons. First, the analyses of both question one and question two are exploratory in nature. Therefore, results that are not significant at a Bonferroni corrected level, but suggest differences of a smaller magnitude may nevertheless be interesting for future research to explore. Second, the Bonferroni correction is quite conservative and thus may dismiss some findings with small effect sizes, which may nonetheless be interesting to examine and may in fact represent actual differences. Third, due to the sample size of at most 61 , there is a greater chance of committing type II error, than in larger samples. The sample size leads to limitations in the power to detect small or even moderate effects. Thus, the second element of the analysis interpretation strategy was to utilize those trend level findings as indicators of a potential difference and therefore a springboard for future research. This two-prong strategy balanced cautious interpretation of findings without neglecting what may be fruitful exploration. 
Results

Analysis of Therapist Adherence: Therapist TLIPT Purity

As a measure of therapist adherence to TLIPT, therapist and observer ratings of the therapist's prescribed and proscribed interventions use was examined. Following the procedure used by Luborsky, McLellan, Woody, O’Brien, and Auerbach (1985), purity was calculated as a ratio of 6 interventions prescribed by TLIPT to a total of 12 interventions that included both the 6 prescribed interventions and 6 proscribed interventions. Perfect adherence is indicated by a purity ratio of 1.0 (i.e., a score of 0 on proscribed interventions). The mean purity ratio as rated by therapists was 0.67 (SD = 0.05; $\mathrm{n}=56$ because five clients had missing data on this measure). The mean purity ratio as rated by observers was $0.66(\mathrm{SD}=0.07, \mathrm{n}=56$ because five clients had missing data on this measure). This suggested a flexible following of the interventions prescribed by the approach. Salsman et al. (2002) found preliminary evidence that a purity level of approximately 0.7 was associated with more improvement on the OQ45 than either higher or lower purity levels.

Analysis of Study Question One: Do clients experience both symptom change and interpersonal change when treated with TLIPT?

\section{Symptom Change in TLIPT}

In order to examine symptom change in TLIPT clients, a series of four repeated measures analysis of variance (ANOVA) was applied to clients’ first and final session OQ45 data. In order to control alpha inflation, a Bonferroni correction was applied to these analyses. Specifically, an alpha of .05 was divided by the four analyses to set a corrected alpha of .0125. The ANOVA revealed that in the OQ Total score there were 
significant reductions over the course of therapy, $F(1,58)=34.36, p<.001, \mathrm{n}=59$.

Table 2 shows the means and standard deviations for the first measure and final measure of all outcome variables. The authors of the OQ45 have applied procedures designed by Jacobson and Truax (1991) to determine cut-off scores and reliable change indices (RCI) for OQ45 scores, which indicate changes required for clinical significance. The cut-off scores can be interpreted as the point at which a person becomes more like those in a non-patient sample than someone in a patient sample. The RCI may be interpreted as the amount of change that needs to occur for the change to be considered reliable and consistent. The cut-off score for the OQ45 Total is 63 and the RCI is 14 points. While the mean first session score was above the cut-off point, the final score was below the cut-off point and the change was greater than 14 points. Thus, this indicates that clients treated with TLIPT tended to start in a range similar to other clinical samples and end therapy more like non-patient samples. Additionally, since the reduction was at a level greater than the RCI, these findings also indicate that the reductions in their symptoms appear to be reliable and consistent.

Further analyses were conducted on the subscales of the OQ45. Repeated measures ANOVAS showed that there were significant reductions in clients' symptom distress subscale scores over the course of therapy, $F(1,59)=42.57, p<.001, \mathrm{n}=60$. The cut-off score for the OQ symptom distress subscale is 36 and the RCI is 10 points. While the mean first session OQ symptom distress score was above the cut-off point, the final score was below the cut-off point and the change was greater than 10 points. Repeated measures ANOVAS also revealed that there were significant reductions in 


\section{Table 2}

Descriptive Statistics for Outcome Variables

\begin{tabular}{|c|c|c|}
\hline Outcome Variable: $(n)$ & $\begin{array}{l}\text { First Measurement } \\
\text { Mean }(S D)\end{array}$ & $\begin{array}{l}\text { Final Measurement } \\
\text { Mean }(S D)\end{array}$ \\
\hline \multicolumn{3}{|l|}{ OQ45: } \\
\hline Total (59) & $76.64(24.32)$ & $56.38(26.85)$ \\
\hline Symptom Distress (60) & $44.28(17.01)$ & $30.60(17.67)$ \\
\hline Interpersonal Relations (60) & $19.45(5.61)$ & $16.00(6.58)$ \\
\hline Social Roles (59) & $13.49(5.36)$ & 10.59 (5.37) \\
\hline Distress (53) & $1.36(0.57)$ & $1.17(0.62)$ \\
\hline Vector Length (54) & $11.77(5.75)$ & $13.22(5.42)$ \\
\hline Affiliation (54) & $-3.03(8.14)$ & $-3.57(8.72)$ \\
\hline Control (54) & $-1.90(9.75)$ & $-2.91(10.47)$ \\
\hline \multicolumn{3}{|l|}{ Therapist on Client IMI: } \\
\hline Vector Length (61) & $13.70(5.94)$ & $12.02(5.23)$ \\
\hline Affiliation (61) & $-3.19(7.39)$ & $-0.30(7.98)$ \\
\hline Control (61) & $-10.39(7.17)$ & $-8.49(6.11)$ \\
\hline \multicolumn{3}{|l|}{ Observer on Client IMI: } \\
\hline Vector Length (53) & $13.40(6.64)$ & $12.91(6.18)$ \\
\hline Affiliation (53) & $-3.98(7.52)$ & $-4.01(7.88)$ \\
\hline Control (53) & $-8.49(8.99)$ & $-8.39(7.61)$ \\
\hline \multicolumn{3}{|l|}{ Significant Other on Client IMI: } \\
\hline Vector Length (24) & $10.64(5.36)$ & $10.42(4.45)$ \\
\hline Affiliation (25) & $-1.62(6.48)$ & $1.30(5.43)$ \\
\hline Control (25) & $-7.81(6.68)$ & $-8.78(4.50)$ \\
\hline \multicolumn{3}{|l|}{ Client IMI-GO: } \\
\hline Vector Length (43) & $11.43(5.70)$ & $10.19(4.75)$ \\
\hline Affiliation (43) & $0.86(5.51)$ & $2.58(5.09)$ \\
\hline Control (43) & $-9.40(7.51)$ & $-7.44(6.19)$ \\
\hline
\end{tabular}


clients' interpersonal relations subscale scores over the course of therapy, $F(1,59)=$ 16.09, $p<.001, \mathrm{n}=60$. The cut-off score for the OQ interpersonal relations subscale is 15 and the RCI is 8 points. The mean first session OQ interpersonal relations score was above the cut-off point. The final OQ interpersonal relations score approached the cutoff point although it did not reach it. The change was not greater than the RCI of 8 points. Finally, repeated measures ANOVAS showed that there were significant reductions in clients' social roles subscale scores over the course of therapy, $F(1,58)=$ 13.67, $p<.001, \mathrm{n}=59$. The cut-off score for the OQ social roles subscale is 12 and the RCI is 7 points. While the mean first session OQ social roles score was above the cut-off point, the final score was below the cut-off point. The amount of change did not reach the RCI level. Overall, support was found for the hypothesis that clients participating in TLIPT would have reductions in symptoms.

Interpersonal Distress Change in TLIPT

Repeated measures ANOVA was also applied to clients’ first and final IIP distress scores. This analysis revealed that clients experienced significant reductions in interpersonal distress over the course of therapy, $F(1,52)=5.37, p<.05, \mathrm{n}=53$. Woodward, Murrell, and Bettler (in press) established an IIP distress score of 1.19, as a cut score above which suggests psychological maladjustment. In this sample, the mean IIP distress score was above this cut score at the beginning of therapy, and subsequently fell below this score at the end of therapy. This supports the hypothesis that clients participating in TLIPT would have reductions in interpersonal distress. 


\section{Interpersonal Rigidity and Extremity Change in TLIPT}

Five repeated measures ANOVAs were applied to vector length scores from the

first and final client IIPs, therapist rating the client IMIs, observer rating the client IMIs, significant other rating the client IMIs, and client rating self IMI-GOs. To control for alpha inflation within this set of analyses, a Bonferroni correction was used for alpha. Since five tests were planned, a corrected alpha of .05 divided by five, or .01 was used. None of the five measures showed significant changes over the course of TLIPT including, IIP vector length, $F(1,53)=2.29, p>.05, \mathrm{n}=54$, observer rating the client IMI, $F(1,52)=0.29, p>.05, \mathrm{n}=53$, and significant other rating the client IMI, $F(1,23)$ $=0.08, p>.05, \mathrm{n}=24$. Vector length on the IMI-GO, however approached significance at the level of a trend (i.e., $F(1,41)=5.06, p=.03, \mathrm{n}=42)$. Additionally, therapist ratings of the client on the IMI approached significance with reductions in vector length over the course of therapy at the level of a trend (i.e., $F(1,60)=4.47, p=.04, \mathrm{n}=61$ ). Thus, these findings only provide minimal support for the hypothesis that in general clients treated with TLIPT experienced reductions in rigidity and extremity of interpersonal patterns as rated by two different sources: self and therapist.

\section{Interpersonal Affiliation Change in TLIPT}

Five repeated measures ANOVAs were applied to affiliation dimension scores from the first and final client IIPs, therapist rating the client IMIs, observer rating the client IMIs, significant other rating the client IMIs, and client rating self IMI-GOs. To control for alpha inflation within this set of analyses a Bonferroni correction was used for alpha. Since five tests were planned, a corrected alpha of .05 divided by five, or .01 was used. Four of these measures showed no significant changes over the course of TLIPT 
including, IIP affiliation $F(1,53)=0.32, p>.05, \mathrm{n}=54$ and observer rating the client IMI affiliation $F(1,52)=0.00, p>.05, \mathrm{n}=53$. Significant other ratings of clients’ affiliation on the IMI showed increases in friendliness that approached significance at the level of a trend, however did not reach the Bonferroni corrected level of significance, (i.e., $F(1,24)=4.55, p=.04, \mathrm{n}=25)$. Client IMI-GO affiliation ratings also showed increases in friendliness that approached significance at the level of a trend, however did not reach the Bonferroni corrected level of significance, (i.e., $F(1,42)=6.00, p=.02$, $\mathrm{n}$ =43). Therapist rating client IMIs showed significant increases in affiliation (i.e., reporting the clients' style of interpersonal interaction was more friendly) over the course of therapy, $F(1,60)=7.25, p<.01, \mathrm{n}=61$. This indicates that therapists reported increases in clients' interpersonal friendliness over the course of TLIPT. Significant others and clients also reported modest increases in affiliation at the level of a trend. Thus, these findings provide some support for the hypothesis that clients treated with TLIPT will have increases in affiliation, i.e. they will become more friendly and less hostile, as rated by three different sources: significant other, self, and therapist.

\section{Interpersonal Control Change in TLIPT}

Five repeated measures ANOVAs were applied to interpersonal control dimension scores from the first and final client IIPs, therapist rating the client IMIs, observer rating the client IMIs, significant other rating the client IMIs, and client rating self IMI-GOs. To control for alpha inflation within this set of analyses a Bonferroni correction was used for alpha. Since five tests were planned, a corrected alpha of .05 divided by five, or .01 was used. Four of these measures showed no significant changes over the course of TLIPT including, IIP control dimension score $F(1,53)=0.89, p>.05$, 
$\mathrm{n}=54$, observer rating the client $\operatorname{IMI} F(1,52)=0.01, p>.05, \mathrm{n}=53$, and significant other rating the client IMI $F(1,24)=0.84, p>.05, \mathrm{n}=25$. Therapist rating the client IMIs showed increases in the control dimension (i.e., indicating increases in dominance) over the course of therapy at the level of a trend, however these scores did not reach the Bonferroni corrected level of significance, $F(1,60)=4.22, p=.044, \mathrm{n}=61$. Client ratings on the IMI-GO indicated significant increases in control dimension score (i.e., indicating increases in dominance and decrease in submissiveness), $F(1,42)=10.64, p<$ $.01, \mathrm{n}=43$. Thus, these findings provide some support for the hypothesis that clients treated with TLIPT will have increases in dominance over the course of therapy as rated by two different sources: self and therapist.

Table 3 summarizes of the results of study question one. All of the significant findings are in the direction predicted by interpersonal theory. Additionally, the findings supporting that clients have decreased interpersonal rigidity and extremity, increased dominance, and increased affiliation were indicated by multiple and distinct sources (i.e., self, therapist, and significant other).

Analyses of Study Question Two: What Factors Contribute to Interpersonal Changes in

\section{Clients Treated with TLIPT?}

The next step in the study was to explore the factors that may contribute to interpersonal changes. The analyses of study question one provided support at the trend level or better that interpersonal changes occurred in clients treated with TLIPT in three areas: rigidity and extremity, affiliation, and control. Although available data included five measures of each of these three areas (i.e., 15 total measures), further analyses were 


\section{Table 3}

Summary of Results for Study Question One

\begin{tabular}{|c|c|c|c|}
\hline Hypothesis & Measure & $\begin{array}{l}\text { Do findings support } \\
\text { the hypothesis? }\end{array}$ & $\begin{array}{l}\text { Statistical } \\
\text { significance } \\
\text { of the finding }\end{array}$ \\
\hline
\end{tabular}

(1) TLIPT clients will have decreased:

a. symptoms

$\begin{array}{lcc}\begin{array}{l}\text { OQ45 } \\ \text { Total }\end{array} & \text { Yes } & p<.001^{* *} \\ \begin{array}{c}\text { Symptom } \\ \text { Distress }\end{array} & \text { Yes } & \\ \begin{array}{c}\text { OQ45 Interpersonal } \\ \text { Relations }\end{array} & \text { Yes } & p<.001^{* *} \\ \text { OQ45 Social Roles } & \text { Yes } & p<.001^{* *} \\ & & p<.001^{* *} \\ \text { IIP distress } & \text { Yes } & p<.05^{* *} \\ \text { lity } & & \\ \text { IIP } & \text { No } & p>.05 \\ \text { Observer IMI } & \text { No } & p>.05 \\ \text { Significant Other IMI No } & p>.05 \\ \text { Therapist IMI } & \text { Yes } & p<.05^{*} \\ \text { IMI-GO } & \text { Yes } & p<.05^{*}\end{array}$

(2) TLIPT clients will

b. interpersonal distress

c. interpersonal rigidity and extremity

have increased:

a. friendliness on affiliation

IIP

No

$p>.05$

Observer IMI No

$p>.05$

Significant Other IMI Yes

$p<.05^{*}$

Therapist IMI

Yes

$p<.01^{* *}$

b. dominance on

IMI-GO

Yes

$p<.05^{*}$

control

IIP

No

$p>.05$

Observer IMI

No

$p>.05$

Significant Other IMI No

$p>.05$

Therapist IMI

Yes

$p<.05^{*}$

IMI-GO

Yes

$p<.01^{* *}$

* Support at the level of a statistical trend

** Support at a statistically significant level 
restricted to only those measures that showed some indications of change over the course of TLIPT. This restriction was put in place to focus further analysis on only those measures that show promise as indicators of change in TLIPT and to avoid the inclusion of measures that may interfere with the clarity of further analyses. Analyses from question one indicated that TLIPT clients tended to have reductions in interpersonal rigidity and extremity on the vector length measures of the IMI-GO and therapist ratings of the client on the IMI. Analyses also indicated that TLIPT clients tended to experience increases in interpersonal affiliation (i.e., increased friendliness and decreased hostility) on the IMI-GO, significant other ratings of the client on the IMI, and therapist ratings of the client on the IMI. Finally, analyses indicated that TLIPT clients tended to experience increases in interpersonal control (i.e., increased dominance and decreased submissiveness) on the IMI-GO and therapist ratings of the client on the IMI.

Therefore, these seven measures (i.e., two for extremity and rigidity, three for affiliation, and two for control) were utilized in further analyses to study question two.

Analyses for question two also required the development of measures of the hypothesized contributors to the interpersonal change process. As indicated by previous research, the contributions of six variables to interpersonal change were explored with these analyses. Measures of early alliance, early symptom reductions, early control complementarity, early affiliation complementarity, later control complementarity, and later affiliation complementarity were developed and utilized in these analyses. Development of Interpersonal Change Groups

Although analyses could be conducted separately on all seven measures showing interpersonal change, this approach would have problems. In particular, this would 
increase the chances of finding spurious significant results due to alpha inflation. In order to control for the number of variables being analyzed, it was decided that analyses would be conducted to group those clients with the most similar profiles of interpersonal change within the three measures of interpersonal patterns (i.e., rigidity and extremity, affiliation, and control). Analyses of question one suggested that changes on interpersonal measures were tending to occur in the same direction across the measures. For example, analyses indicated that clients tended to have increases in dominance on both the IMI-GO and therapist ratings of the client on the IMI, rather than increasing on one measure while decreasing on the second. Therefore, analyses were conducted to determine groups of clients with high change in the indicated direction versus no change or change in the opposite direction for each of the three measures of interpersonal patterns. In other words, analyses were utilized to categorize all clients into two separate groups for each of the three measures of interpersonal patterns: 1) those with relatively large reductions in rigidity and extremity versus those with small reductions or increases in rigidity and extremity (see below for how these were calculated), 2) those with relatively large increases in friendliness versus those with small increases or decreases in friendliness, and 3) those with relatively large increases in dominance versus those with small increases or decreases in dominance.

In order to define groups, variables indicating interpersonal change had to be calculated. A residual change method of calculating interpersonal change was used, as it has been indicated as superior to the use of simple difference scores in measuring change (Willett, 1988). Simple change scores (i.e., calculating a client’s change by subtracting each client's score at the beginning of therapy from his or her score at the end or therapy) 
do not account for the clients' varying number of sessions. Past research (e.g., Kopta et al., 1994; Schauenburg et al., 2000) has indicated that length of treatment may be an important determinant in bringing about interpersonal change, that is, more sessions may lead to better outcomes. In order to control for length of treatment, a residual change method was used for all seven measures. The residual change method utilized regression analyses, which predict final measurement scores (i.e., the dependent variable) using the total number of sessions and initial score on the measure as independent variables. As a result of the regression analysis, a residual score is retained for each client. The residual scores represents the degree to which the final score on a given outcome measure is higher (i.e., positive scores) or lower (i.e., negative scores) than what is predicted when controlling for initial score and number of sessions. Thus, negative residuals for vector length measures indicate large reductions in rigidity and extremity over the course of therapy, positive residuals for the affiliation dimension measures indicate large increases in friendliness, and positive residuals for the control dimension indicate large increases in dominance. These residual scores were subsequently used as the parameters for defining groups through the use of K-means cluster analyses.

Three separate K-means cluster analyses were used to define distinct groups. Kmeans cluster analysis is a procedure that is used to group individuals into relatively homogenous groups based on specified variables. For the first K-means analysis, the specified variables were the residual change scores for vector length on the IMI-GO and the therapist rating of the client on the IMI. For the second K-means analysis, the specified variables were the residual change scores for the affiliation dimension on the IMI-GO, the significant other rating of the client on the IMI, and the therapist rating of 
the client on the IMI. For the third K-means analysis, the specified variables were residual change scores for the control dimension on the IMI-GO and the therapist rating of the client on the IMI. Each analysis required that the number of clusters be specified prior to running the analysis. Based on the findings from question one, two clusters were specified for each of the three analyses. That is, all clients with available data were categorized into two groups on three separate occasions. The algorithm for K-means cluster analysis attempts to maximize differences among the specified number of groups based on the parameters. Therefore, the analyses statistically separated the sample to find maximally different groups of rigidity and extremity change, affiliation change, and control change. Additionally, pairwise rather than listwise deletion of data was used in the K-means cluster analyses. This option provides the advantage of using all available data for all clients, rather than only including those clients with data available on all parameters.

\section{Calculation of Variables Hypothesized to Contribute to Interpersonal Change}

Measures of early alliance, early symptom reductions, early control complementarity, early affiliation complementarity, later control complementarity, and later affiliation complementarity were calculated to be used in further analyses. For the purposes of these analyses the early phase of therapy was considered to be sessions one through four. This period was chosen for several reasons. First of all, the CMP case conceptualization meeting was scheduled to occur after session four. At this meeting, the client's interpersonal pattern is conceptualized, and subsequently suggestions for how the therapist may disengage from, or provide a non-complementary response to the client's pattern are indicated. Thus, after the fourth session the therapist is encouraged to 
deliberately move from being highly complementary to the client's interpersonal pattern to being less complementary, thus moving into the middle phase of therapy. Secondly, studies which have examined stages of complementarity in therapy (Tracey, 1987; Tracey et al., 1999), the contribution of alliance to outcome (Gaston, 1990; Hartley \& Strupp, 1983; Horvath \& Luborsky, 1993; Lambert, 1992), and early symptom reductions (Gaston et al. 1991) have been consistent with considering the first four sessions as the beginning stage of therapy.

Thus, measures of alliance and symptom reduction in the early phase of therapy, which are predicted to contribute to interpersonal changes, were calculated using data from sessions one through four. The early alliance measure was calculated by taking the mean WAI total score for sessions one through four. The measure of early symptom reduction was calculated using the residual change method for sessions one and four OQ45 total score. The residual change method utilized regression analyses, which predict session four OQ45 total score using session one OQ45 total score as the independent variable. As a result of the regression analysis, a residual score was retained for each client. The residual scores represent the degree to which session four OQ45 total score was higher (i.e., positive scores) or lower (i.e., negative scores) than what was predicted when controlling for session one total score. Thus, negative residuals indicate large symptom reductions in the early phase of therapy and positive residuals indicate smaller than expected reductions or increases in symptoms in the early phase of therapy.

Early complementarity measures were calculated using data from sessions one and four. A measure of initial complementarity between the therapist and the client on the control dimension was calculated by subtracting the control dimension score for the 
client's self-rated IMI-GO after the first session from the control dimension score for the therapist's self-rated IMI-GO after the first session. The absolute difference indicates complementarity on control. Disregarding the sign of the difference removed the impact of the order of subtraction (i.e., therapist minus client or vice versa) on complementarity scores. This measure examines the complementarity of client and therapist perceptions of how others view their typical interpersonal pattern at the start of therapy (i.e., through the use of IMI-GO ratings). A larger difference on this initial control complementarity indicates greater complementarity, as a large difference will indicate that one person is reporting a more dominant interpersonal style while the other is reporting a more submissive style. A second measure of early complementarity was also calculated in similar fashion using session four therapist ratings of clients' control dimension on the IMI and client rating of the therapists' control dimensions on the IMI. The mean of the session one IMI-GO complementarity and session four IMI complementarity measures served as the measure of total early control complementarity. The mean of these two measures was used to get a representative sample of complementarity in the early phase of therapy. The session one IMI-GO ratings provided perspectives of how the clients and therapists view the complementarity of their typical general interpersonal patterns at the beginning of therapy, while the session four ratings provide perspectives of clients' and therapists' in-session complementarity within the beginning stage of therapy. Similar to the procedures for early control complementarity, session one client IMI-GO affiliation dimension score and session one therapist IMI-GO affiliation dimension score were used to calculate one measure of early affiliation complementarity. Session four therapist's rating of client's affiliation dimension score on the IMI and client's rating of the 
therapist's affiliation dimension score on the IMI were used to calculate a second measure of early affiliation complementarity. The mean of these two measures was utilized as a measure of total early affiliation complementarity. The main difference between control complementarity and affiliation complementarity is that a large difference on early affiliation complementarity indicates low complementarity, as a large difference will be indicative that one person is friendly while the other person is more hostile.

Later complementarity scores were calculated using measures from sessions five, seven, and nine. Later complementarity scores were calculated with IMIs beginning with session five because this is generally the time when the therapist conceptualizes the client's CMP, and thus theoretically moves from the beginning stage of therapy to the middle stage of therapy. Data from sessions seven and nine were also used to provide multiple indicators of middle stage complementarity. Session nine data was not used for calculating later complementarity for those clients with only nine sessions $(n=5)$, as session nine measures for these clients were utilized in the calculation of outcomes. In these cases, the mean therapist and client complementarity was calculated using data only from sessions five and seven. The measures used in calculating complementarity were therapists’ IMI ratings of the clients and clients’ IMI ratings of the therapists. These measures were calculated using identical procedures as described for early complementarity measures except using sessions five, seven, and nine therapists' ratings of clients’ control and affiliation scores and clients’ ratings of therapists' control and affiliation scores. The mean of session five, seven, and nine control complementarity was utilized as a measure of later mean control complementarity. The mean of session 
five, seven, and nine affiliation complementarity was utilized as a measure of later mean affiliation complementarity.

Analysis of Complementarity from the Beginning to the Middle Stage

In order to test the hypothesis that TLIPT therapists would actually engage in lower complementarity in the middle stage than in the beginning stage, two repeated measures ANOVAs were conducted. These repeated measures ANOVAs examined if there were reductions in control and affiliation complementarity from the beginning to the middle stage of therapy. The mean of the early control complementarity was 8.08 with a standard deviation of 5.49, while the mean of the later control complementarity was 7.18 with a standard deviation of 4.08 (note: smaller control complementarity numbers indicate less complementarity). The ANOVAs examining total early control complementarity and later total control complementarity revealed that there was not a significant change in control complementarity from the early stage to the middle stage of therapy, $F(1,44)=0.80, p>.05, \mathrm{n}=45$. This did not support the hypothesis that TLIPT client and therapist dyads experience reductions in control complementarity from the early to the middle phase of therapy. The mean of the early affiliation complementarity was 6.69 with a standard deviation of 3.97, while the mean of the later affiliation complementarity was 9.06 with a standard deviation of 5.57 (note: larger affiliation complementarity numbers indicate less complementarity). The ANOVAs examining total early affiliation complementarity and later total affiliation complementarity revealed that there was a significant reduction in affiliation complementarity from the early stage of therapy to the middle stage of therapy, $F(1,44)=4.34, p<.05, \mathrm{n}=45$. This supports 
the hypothesis that TLIPT client and therapist dyads would experience significant reductions in affiliation complementarity from the early to the middle phase of therapy. K-means Cluster Analysis of Rigidity and Extremity Parameters

The first K-means analysis, clustered 61 clients based on their residual change scores calculated using their first and final vector length on the IMI-GO and on the therapists' ratings of the client on the IMI. That is, residual change score for clients' selfreport IMI-GO vector length and residual change scores for therapists' ratings of clients' vector length were entered as the sorting parameters for the first K-means cluster analysis. This resulted in cluster one with 30 clients and cluster two with 31 clients. It was expected that one group would include individuals with large reductions in rigidity and extremity and the second group would include individuals with small reductions or increases. Table 4 includes the mean residual change scores across all clients, for those in cluster one, and those in cluster two. Due to the way they are calculated, the mean of any set of residual change scores is always zero. Additionally, the signs (i.e., positive or negative) of residual change scores do not necessarily indicate whether a score has increased or decreased overall, but instead indicate whether a client's actual vector length score has changed more or less than what the regression equation predicts. Because of the potential for confusion in interpreting residual change scores, simple change scores are also included in Table 4 to provide descriptive statistics of gross overall changes. Table 4 shows that individuals in cluster one for this set of analyses had an average decrease in therapists' ratings of the client's vector length on the IMI of 4.24, which is a 


\section{Table 4}

Means of Vector Length Variables among K-Means Vector Length Clusters

\begin{tabular}{|c|c|c|c|}
\hline Variable & $\begin{array}{l}\text { "Large Change" } \\
\text { Cluster } 1\end{array}$ & $\begin{array}{l}\text { "Small Change" } \\
\text { Cluster } 2\end{array}$ & Total \\
\hline \multicolumn{4}{|c|}{ Therapist rating Client IMI Vector } \\
\hline \multicolumn{4}{|c|}{ Length: } \\
\hline \multicolumn{4}{|c|}{ Residual Change Score } \\
\hline$M$ & -0.56 & 0.54 & 0 \\
\hline$S D$ & 0.75 & 0.88 & 0.98 \\
\hline$n$ & 30 & 31 & 61 \\
\hline \multicolumn{4}{|c|}{ Simple Change Score } \\
\hline$M$ & -4.24 & 0.80 & -1.68 \\
\hline$S D$ & 5.96 & 5.43 & 6.20 \\
\hline$n$ & 30 & 31 & 61 \\
\hline \multicolumn{4}{|c|}{ IMI-GO Vector Length: } \\
\hline \multicolumn{4}{|c|}{ Residual Change Score } \\
\hline$M$ & -0.55 & 0.66 & 0 \\
\hline$S D$ & 0.84 & 0.68 & 0.98 \\
\hline$n$ & 23 & 19 & 42 \\
\hline \multicolumn{4}{|c|}{ Simple Change Score } \\
\hline$M$ & -2.99 & 0.87 & -1.24 \\
\hline$S D$ & 3.36 & 2.60 & 3.58 \\
\hline$n$ & 23 & 19 & 42 \\
\hline
\end{tabular}


decrease of 0.71 standard deviations. Individuals in cluster one also had a mean decrease in self rated IMI-GO vector length of 2.99, which is a decrease of 0.89 standard deviations. Thus, cluster one matches what may be described as large reductions in rigidity and extremity, and is labeled as the "large change" cluster. Individuals in cluster two had an average increase in therapists' ratings of the client's vector length on the IMI of 0.80 , which is an increase of 0.15 standard deviations. Individuals in cluster two also had an average increase in IMI-GO vector length of 0.87 , which is an increase of 0.33 standard deviations. Thus, cluster two matches what may be described as stable or small increases in rigidity and extremity, and is labeled as the "small change" cluster. Comparisons of The Two Rigidity and Extremity Clusters

A series of six one-way ANOVAs were conducted to compare the two rigidity and extremity clusters. These ANOVAs were tests of hypotheses that those with large reductions in rigidity and extremity would have higher early alliance, more early symptom reductions, higher early control complementarity, higher early affiliation complementarity, lower later control complementarity, and lower later affiliation complementarity than those with stable or small increases in rigidity and extremity. Table 5 contains the means and standard deviations of these scores for those in cluster one and those in cluster two. In order to control alpha inflation a Bonferroni correction was applied to these analyses. Specifically, an alpha of .05 was divided by six analyses to set a corrected alpha of .0083. These ANOVAs revealed that there were no significant differences between the two clusters at the Bonferroni corrected level. However, those with greater reductions in rigidity and extremity over the course of therapy had some indication of higher early control complementarity at a level 


\section{Table 5}

Means of Therapy Process Variables among K-Means Vector Length Clusters

\begin{tabular}{l}
\hline Variable \\
\hline Early Alliance
\end{tabular}

$M$

SD

$n$

Early Symptom Reduction

Standardized Residual

$M$

$S D$

$n$

Total Early Control

Complementarity $^{\mathrm{a}}$

$M$

$S D$

$n$

Total Early Affiliation

Complementarity $^{\mathrm{a}}$

$M$

$S D$

$n$

Total Later Control

Complementarity $^{\mathrm{a}}$

$M$

$S D$

$n$

Total Later Affiliation

Complementarity $^{\mathrm{a}}$

$M$

$S D$

$n$
65.15

8.74

30

0.04

1.08

28

9.53

5.84

26

7.29

4.30

26

7.38

4.09

29

8.33

5.42

29
68.08

9.56

31

$-0.14$

1.13

26

1.10

54

9.21

61

Total

66.63

$-0.05$

6.68

8.08

4.84

27

5.49

53

6.69

3.97

52

$\begin{array}{ll}3.60 & 3.97 \\ 26 & 52\end{array}$

6.94

7.18

4.15

4.08

24

53

9.91

9.06

5.73

5.57

25

\footnotetext{
${ }^{\mathrm{a}}$ Higher numbers for the control complementarity measures indicate greater

complementarity, whereas higher numbers for the affiliation complementarity measures indicate lower degrees of complementarity.
} 
approaching a trend $(F(1,51)=3.75, p=.058, \mathrm{n}=52)$. This provides limited support for future exploration of the hypothesis that high early control complementarity may be associated with greater reductions in rigidity and extremity.

\section{K-means Cluster Analysis of Affiliation Parameters}

For the second K-means analysis, the variables used as parameters were residual change scores for the affiliation dimension on the IMI-GO, significant others' ratings of the client on the IMI, and therapists' ratings of the client on the IMI. This resulted in cluster one with 35 clients and cluster two with 26 clients. It was expected that one group would include individuals with large increases in affiliation (i.e., increased friendliness) and the second group would include individuals with small increases or reductions. Table 6 includes the mean residual change and simple change scores across total clients and separated for those in cluster one and those in cluster two. Individuals in cluster one for this set of analyses had an average increase in therapists' ratings of the client's affiliation on the IMI of 7.30, which is an increase of 0.94 standard deviations. Individuals in cluster one also had an average increase in significant others' affiliation ratings of 4.15, which is an increase of .65 standard deviations. Finally, individuals in cluster one had an average increase in IMI-GO affiliation of 0.92, which is an increase of 0.22 standard deviations. However, the increase in IMI-GO affiliation for cluster one is smaller than the increase in affiliation observed on the IMI-GO in cluster two. Thus, cluster one matches what may be described as moderate to large increases in friendliness, and is labeled as the "large change" cluster. Table 6 shows that individuals in cluster two of this set of analyses had an average decrease in therapists' ratings of the client's affiliation on the IMI of 3.03, which is a decrease of 0.62 standard deviations. Individuals in 


\section{Table 6}

$\underline{\text { Means of Affiliation Variables among K-Means Affiliation Clusters }}$

\begin{tabular}{|c|c|c|c|}
\hline Variable & $\begin{array}{l}\text { "Large Change” } \\
\text { Cluster (1) }\end{array}$ & $\begin{array}{l}\text { "Small Change" } \\
\text { Cluster (2) }\end{array}$ & Total \\
\hline \multicolumn{4}{|c|}{ Therapist rating Client IMI } \\
\hline \multicolumn{4}{|l|}{ Affiliation: } \\
\hline \multicolumn{4}{|c|}{ Residual Change Score } \\
\hline$M$ & 0.65 & -0.87 & 0 \\
\hline$S D$ & 0.66 & 0.59 & 0.98 \\
\hline$n$ & 35 & 26 & 61 \\
\hline \multicolumn{4}{|c|}{ Simple Change Score } \\
\hline$M$ & 7.30 & -3.03 & 2.89 \\
\hline$S D$ & 7.75 & 4.84 & 8.39 \\
\hline$n$ & 35 & 26 & 61 \\
\hline \multicolumn{4}{|c|}{$\begin{array}{l}\text { Significant Other rating Client IMI } \\
\text { Affiliation: } \\
\text { Residual Change Score }\end{array}$} \\
\hline$M$ & 0.32 & -1.26 & 0 \\
\hline$S D$ & 0.65 & 1.00 & 0.96 \\
\hline$n$ & 20 & 5 & 25 \\
\hline \multicolumn{4}{|c|}{ Simple Change Score } \\
\hline$M$ & 4.15 & -1.97 & 2.93 \\
\hline$S D$ & 6.43 & 7.00 & 6.86 \\
\hline$n$ & 20 & 5 & 25 \\
\hline \multicolumn{4}{|l|}{ IMI-GO Affiliation: } \\
\hline \multicolumn{4}{|c|}{ Residual Change Score } \\
\hline$M$ & -0.11 & 0.17 & 0 \\
\hline$S D$ & 0.99 & 0.96 & 0.98 \\
\hline$n$ & 26 & 17 & 43 \\
\hline \multicolumn{4}{|c|}{ Simple Change Score } \\
\hline$M$ & 0.92 & 2.94 & 1.72 \\
\hline$S D$ & 4.25 & 4.98 & 4.60 \\
\hline$n$ & 26 & 17 & 43 \\
\hline
\end{tabular}


cluster two also had a mean decrease in significant others' ratings of clients' affiliation on the IMI of 1.97 , which is a decrease of 0.28 standard deviations. Finally, individuals in cluster two had an average increase in self rated IMI-GO affiliation of 2.94, which is an increase of 0.59 standard deviations. Thus, while cluster two matches what may be described as moderate reductions in friendliness for therapist and significant other ratings of the client, cluster two also includes moderate increases in friendliness as rated by the client on the IMI-GO. This cluster is labeled as the "small change" cluster.

\section{Comparisons of The Two Affiliation Clusters}

A series of six one way ANOVAs were conducted to compare the two affiliation clusters. These ANOVAs were tests of hypotheses that those with high increases in friendliness would have higher early alliance, more early symptom reductions, higher early control complementarity, higher early affiliation complementarity, lower later control complementarity, and lower later affiliation complementarity than those with some decreases in affiliation. Table 7 contains the means and standard deviations of these scores for those in cluster one and those in cluster two. In order to control alpha inflation a Bonferroni correction was applied to these analyses. Specifically, an alpha of .05 was divided by six analyses to set a corrected alpha of .0083. These ANOVAs revealed that there were no significant differences between the two clusters at the Bonferroni corrected level. However, individuals in cluster one (i.e., those with moderate to large increases in overall affiliation) had higher later affiliation complementarity at the level of a statistical trend $(F(1,52)=4.90, p=.031, \mathrm{n}=53)$. This finding runs contrary to the hypothesis that lower complementarity in the later stage of therapy would be associated with greater interpersonal changes. 


\section{Table 7}

Means of Therapy Process Variables among K-Means Affiliation Clusters

\begin{tabular}{|c|c|c|c|}
\hline Variable & $\begin{array}{l}\text { "Large Change” } \\
\text { Cluster (1) }\end{array}$ & $\begin{array}{l}\text { "Small Change" } \\
\text { Cluster (2) }\end{array}$ & Total \\
\hline \multicolumn{4}{|c|}{ Early Alliance } \\
\hline$M$ & 65.84 & 67.71 & 66.63 \\
\hline$S D$ & 8.16 & 10.52 & 9.21 \\
\hline$n$ & 35 & 26 & 61 \\
\hline \multicolumn{4}{|c|}{ Early Symptom Reduction } \\
\hline$M$ & 0.08 & -0.20 & -0.05 \\
\hline$S D$ & 0.84 & 1.36 & 1.10 \\
\hline$n$ & 30 & 24 & 54 \\
\hline \multicolumn{4}{|c|}{ Total Early Control } \\
\hline$M$ & 8.12 & 8.01 & 8.08 \\
\hline$S D$ & 5.83 & 5.07 & 5.49 \\
\hline$n$ & 32 & 21 & 53 \\
\hline \multicolumn{4}{|c|}{ Total Early Affiliation } \\
\hline$M$ & 6.69 & 6.69 & 6.69 \\
\hline$S D$ & 3.91 & 4.17 & 3.97 \\
\hline$n$ & 32 & 20 & 52 \\
\hline \multicolumn{4}{|c|}{$\begin{array}{l}\text { Total Later Control } \\
\qquad \text { Complementarity }\end{array}$} \\
\hline$M$ & 6.56 & 8.06 & 7.18 \\
\hline$S D$ & 4.06 & 4.03 & 4.08 \\
\hline$n$ & 31 & 22 & 53 \\
\hline \multicolumn{4}{|c|}{$\begin{array}{l}\text { Total Later Affiliation } \\
\text { Complementarity }^{\mathrm{a}}\end{array}$} \\
\hline$M$ & 7.72 & 11.01 & 9.06 \\
\hline$S D$ & 5.23 & 5.58 & 5.57 \\
\hline$n$ & 32 & 22 & 54 \\
\hline
\end{tabular}

${ }^{\mathrm{a}}$ Higher numbers for the control complementarity measures indicate greater

complementarity, whereas higher numbers for the affiliation complementarity measures indicate lower degrees of complementarity. 


\section{K-means Cluster Analysis of Control Parameters}

For the third K-means analysis, the variables used as parameters were residual change scores for the control dimension on the IMI-GO and the therapist rating of the client on the IMI. This resulted in cluster one with 37 clients and cluster two with 24 clients. It was expected that one group would include individuals with large increases in dominance and the second group would include individuals with small increases or reductions. Table 8 includes the mean residual change and simple change scores across all clients and separated for those in cluster one and those in cluster two. Table 8 shows that individuals in cluster one for this set of analyses had an average increase in therapists' ratings of the client's control dimension (i.e., increases in dominance) on the IMI of 4.43, which is an increase of 0.66 standard deviations. Individuals in cluster one also had a mean increase in self rated IMI-GO control of 3.68, which is an increase of 1.12 standard deviations. Thus, cluster one matches what may be described as large increases in dominance, and is labeled as the "large change" cluster. Individuals in cluster two had an average decrease in therapists' ratings of the client's control dimension on the IMI of 1.99, which is a decrease of 0.32 standard deviations. Individuals in cluster two also had an average decrease in IMI-GO control of 0.95, which is a decrease of 0.30 standard deviations. Thus, cluster two matches what may be described as small to moderate reductions in dominance, and is labeled as the "small change” cluster.

\section{Comparisons of The Two Control Clusters}

A series of six one way ANOVAs was conducted to compare the two control clusters. These ANOVAs were tests of hypotheses that those with large increases in dominance 


\section{Table 8}

$\underline{\text { Means of Control Variables among K-Means Control Clusters }}$

\begin{tabular}{clll}
\hline Variable & $\begin{array}{l}\text { "Large Change" } \\
\text { Cluster (1) }\end{array}$ & $\begin{array}{l}\text { "Small Change” } \\
\text { Cluster (2) }\end{array}$ & Total \\
\hline $\begin{array}{c}\text { Therapist rating Client IMI Control: } \\
\text { Residual Change Score }\end{array}$ & & & \\
$M$ & 0.45 & -0.69 & 0 \\
$S D$ & 0.88 & 0.70 & 0.98 \\
$n$ & 37 & 24 & 61 \\
Simple Change Score & & & 1.90 \\
$M$ & 4.43 & -1.99 & 7.23 \\
$S D$ & 6.71 & 6.29 & 61 \\
$n$ & 37 & 24 & \\
IMI-GO Control: & & & 0 \\
Residual Change Score & & & 0.98 \\
$M$ & 0.50 & -0.85 & 42 \\
$S D$ & 0.73 & 0.73 & 1.96 \\
$n$ & 27 & 16 & 3.93 \\
$M$ & & & 43 \\
SD & 3.68 & -0.95 & \\
$n$ & 3.29 & 3.20 &
\end{tabular}


would have higher early alliance, more early symptom reductions, higher early control complementarity, higher early affiliation complementarity, lower later control complementarity, and lower later affiliation complementarity than those with small to moderate reductions in dominance. Table 9 contains the means and standard deviations of these scores for those in cluster one and those in cluster two. In order to control alpha inflation a Bonferroni correction was also applied to these analyses, with an alpha of .05 divided by six analyses, or .0083. These ANOVAs revealed that there were no significant differences between the two clusters at the Bonferroni corrected level. However, those with greater increases in dominance over the course of therapy had higher early control complementarity at the level of a trend $(F(1,51)=5.03, p=.029$, $\mathrm{n}$ =52). This provides support for further exploration of the hypothesis that higher early control complementarity may be associated with later increases in dominance. Table 10 provides a summary of the results for the second study question. 


\section{Table 9}

Means of Therapy Process Variables among K-Means Control Clusters

\begin{tabular}{|c|c|c|c|}
\hline Variable & $\begin{array}{l}\text { "Large Change" } \\
\text { Cluster (1) }\end{array}$ & $\begin{array}{l}\text { "Small Change" } \\
\text { Cluster (2) }\end{array}$ & Total \\
\hline \multicolumn{4}{|c|}{ Early Alliance } \\
\hline$M$ & 65.69 & 68.09 & 66.63 \\
\hline$S D$ & 9.07 & 9.42 & 9.21 \\
\hline$n$ & 37 & 24 & 61 \\
\hline \multicolumn{4}{|c|}{$\begin{array}{l}\text { Early Symptom Reduction } \\
\text { Standardized Residual }\end{array}$} \\
\hline$M$ & -0.05 & -0.04 & -0.05 \\
\hline$S D$ & 1.13 & 1.08 & 1.10 \\
\hline$n$ & 34 & 20 & 54 \\
\hline \multicolumn{4}{|c|}{$\begin{array}{l}\text { Total Early Control } \\
\text { Complementarity }^{\mathrm{a}}\end{array}$} \\
\hline$M$ & 9.35 & 5.99 & 8.08 \\
\hline$S D$ & 6.04 & 3.70 & 5.49 \\
\hline$n$ & 33 & 20 & 53 \\
\hline \multicolumn{4}{|c|}{$\begin{array}{l}\text { Total Early Affiliation } \\
\text { Complementarity }^{\mathrm{a}}\end{array}$} \\
\hline$M$ & 6.69 & 6.67 & 6.69 \\
\hline$S D$ & 4.04 & 3.96 & 3.97 \\
\hline$n$ & 32 & 20 & 52 \\
\hline \multicolumn{4}{|c|}{$\begin{array}{l}\text { Total Later Control } \\
\text { Complementarity }^{\mathrm{a}}\end{array}$} \\
\hline$M$ & 7.79 & 6.09 & 7.18 \\
\hline$S D$ & 4.16 & 3.79 & 4.08 \\
\hline$n$ & 34 & 19 & 53 \\
\hline \multicolumn{4}{|c|}{$\begin{array}{l}\text { Total Later Affiliation } \\
\text { Complementarity }^{\mathrm{a}}\end{array}$} \\
\hline$M$ & 8.92 & 9.33 & 9.06 \\
\hline$S D$ & 5.48 & 5.87 & 5.57 \\
\hline$n$ & 35 & 19 & 54 \\
\hline
\end{tabular}

${ }^{\mathrm{a}}$ Higher numbers for the control complementarity measures indicate greater

complementarity, whereas higher numbers for the affiliation complementarity measures indicate lower degrees of complementarity. 


\section{Table 10}

Summary of Results for Study Question Two

Hypothesis

Do findings support Statistical

the hypothesis? significance

of the finding

(3) From the beginning to the middle of therapy,

TLIPT clients will have decreased:
a. control complementarity
No
$p>.05$
b. affiliation complementarity
Yes
$p<.05^{* *}$

(4) TLIPT clients with larger overall reductions in rigidity and extremity of their interpersonal patterns will have significantly:

a. higher early alliance

No $\quad p>.05$

b. higher early symptom reduction

No

$p>.05$

c. higher early complementarity on the affiliation dimension

No

$p>.05$

d. higher early complementarity on the control dimension

No

$p=.058$

e. lower middle stage complementarity on the affiliation dimension

No

$p>.05$

f. lower middle stage complementarity on the control dimension

No

$p>.05$

than those with stable or increasing patterns of rigidity and extremity.

(5) TLIPT clients with larger overall increases in friendliness on the affiliation dimension will have significantly:

a. higher early alliance

No

$p>.05$

b. higher early symptom reduction

No

$p>.05$

c. higher early complementarity on the affiliation dimension

No

$p>.05$

d. higher early complementarity on the control dimension

No

$p>.05$

e. lower middle stage complementarity on the affiliation dimension

No***

$p<.05$

f. lower middle stage complementarity on the control dimension

No

$p>.05$

than those with stable or decreasing friendliness on the affiliation dimension.

(6) TLIPT clients with larger overall increases in dominance on the control dimension will have significantly:

a. higher early alliance

b. higher early symptom reduction

No

$p>.05$

No

$p>.05$ 
c. higher early complementarity on the affiliation dimension

No

$p>.05$

d. higher early complementarity on the control dimension

Yes

$p<.05^{*}$

e. lower middle stage complementarity on the affiliation dimension

No

$p>.05$

f. lower middle stage complementarity on the control dimension

No

$p>.05$

than those with stable or decreasing dominance on the control dimension.

* Support at the level of a statistical trend

** Support at a statistically significant level

*** Those with larger overall reductions in friendliness on the affiliation dimension had higher middle stage complementarity on the affiliation dimension at the level of a trend. This was contrary to the hypothesis. 


\section{Discussion}

This study sought to help fill the gap in research examining interpersonal change as an outcome of psychotherapy through exploration of two main questions. The first question examined if clients treated with TLIPT experienced reductions in both symptoms and interpersonal changes. Findings provided strong evidence that clients experienced symptom reductions and some support that clients experienced interpersonal changes in some areas. The second question explored some elements that may contribute to interpersonal change. Findings provided only limited suggestion that elements of complementarity in the therapy relationship may warrant further exploration as contributors to interpersonal change.

Study Question One: Do clients experience both symptom change and interpersonal change when treated with TLIPT?

\section{Symptom Reductions}

This study indicated that clients treated with TLIPT experienced significant reductions in a wide variety of symptoms. Although, there was no control group to determine if the symptom reductions were due to the treatment or other factors (e.g., spontaneous remission or regression toward the mean), data from other research provides support that TLIPT contributed to at least some of the reductions. In particular, data from the standardization of the OQ45 (Lambert et al., 1996) provides cut off scores and RCIs that indicate that TLIPT clients experienced statistically significant change that goes beyond what might be expected in individuals who are not in therapy. Clients crossed the cut off scores for the OQ45 total score, symptom distress score, and the social roles scores. These changes indicated that prior to treatment, clients' scores were similar to a 
sample of clinically impaired individuals. Following therapy, clients' scores on these three scales were within a range that may be expected in a psychologically healthy population. Additionally, TLIPT clients' reductions in symptoms on average met the RCI criteria for OQ total and symptom distress scales. This indicates that the changes experienced by the clients on the total score and symptom distress were reliable and go beyond what may be expected to occur by chance.

Data indicated that TLIPT clients tended to have reductions in interpersonal problems over the course of treatment. Although TLIPT clients' OQ interpersonal relations scores, on average, decreased significantly over therapy, the scores did not drop below the cut off score and reductions did not reach the RCI level. However, the average OQ interpersonal relations score at final measurement was only one point above the cut off score. Further evidence of a reduction of interpersonal problems in TLIPT clients comes from the finding that clients experienced a significant reduction in IIP interpersonal distress over the course of therapy. The IIP distress score did drop from above to below the clinically significant cut score over the course of therapy. These findings indicate that clients reported significantly lower interpersonal distress at the end of therapy than at the beginning of therapy. Thus, TLIPT clients experienced significant reductions in interpersonal problems on two measures.

Further support that some of the symptom reductions may be due to TLIPT, is found in a study of the stability of the OQ45 in a non-clinical sample. Lambert et al., (1996) designed the OQ45 to be sensitive to change and found that scores were sensitive to changes over the course of seven therapy sessions in a clinical sample. The authors also found that scores in a sample of undergraduates who were not in therapy had no 
significant changes over the course of seven weeks. Vermeersch, Lambert, and Burlingame (2000) found further support that untreated individuals did not have significant changes in OQ45 scores, while individuals in therapy had significant reductions. Therefore, TLIPT clients' reductions on the OQ45 may go beyond what might be expected in a non-treatment condition.

\section{Interpersonal Changes}

On measures of interpersonal rigidity and extremity, clients showed reductions of vector length scores at trend levels for two measures. In particular, clients' self-report on the IMI-GO and therapists' ratings of the client showed trend level reductions. These findings are consistent with the hypothesis that TLIPT clients have reductions in rigidity and extremity of interpersonal patterns. Reduction of interpersonal rigidity and extremity is a target of TLIPT, as rigidity and extremity indicate that a client is often not adaptive in his or her interpersonal approach. The reductions present in therapists' report of rigidity and extremity may indicate that at least within the therapeutic relationship, clients became less rigid. Reductions in the IMI-GO ratings also may indicate that clients perceived themselves as becoming less rigid and extreme in their general approach to interpersonal interactions. These reductions may be indicative of changes beyond the therapeutic relationship. There was not evidence of a reduction in client IIP, observer, or significant other ratings. Future research should further explore the tentative findings of these statistical trends. Additionally, research may examine if individuals who have reductions in rigidity and extremity in the therapeutic relationship, also reduce their rigidity and extremity in their approach to relationships subsequent to therapy. 
On measures of interpersonal affiliation, clients showed statistically significant increases in friendliness scores on one measure and increases at trend levels for two measures. Therapists reported a statistically significant increase in their ratings of client friendliness, while significant others and clients' self-reports showed trend level increases. These findings are consistent with the hypothesis that TLIPT clients become friendlier on the affiliation dimension over the course of treatment. It is notable that TLIPT clients started out in the hostile range of the control dimension on all of the measures, except for the client self-reported IMI-GO. While this increased friendliness is not necessarily a target of TLIPT, these findings are consistent with previous research indicating that friendliness tends to increase over the course of CBT for generalized anxiety (Constantino, 2003), CBT for depression (Sledge, 1999), and milieu therapy for severely psychiatrically disturbed patients (Granberg \& Armelius, 2003). Additionally, the findings of this study are consistent with previous research that indicated that increased friendliness both in and out of the therapeutic relationship can be beneficial. Studies have indicated that a hostile approach can lead to negative outcomes within therapy (Tasca \& McMullen, 1993) and outside of therapy (Estroff \& Nowicki, 1992), while a more friendly approach tends to lead to better outcomes. The increased friendliness developed by clients over the course of TLIPT may lead to more positive outcomes for individuals outside of therapy.

On measures of interpersonal control, clients showed statistically significant increases in dominance on the self-reported IMI-GO and increases at a trend level for therapists' ratings of clients. These findings are consistent with the hypothesis that TLIPT clients become more dominant on the control dimension over the course of 
treatment. It is notable that clients started and remained within the submissive range of interpersonal behaviors over the course of therapy. Thus, clients experienced increases in interpersonal dominance, but their interpersonal style remained within the submissive range. While increased dominance is not necessarily a target of TLIPT, these findings are consistent with the limited previous research on changes in interpersonal control as a result of therapy. Studies have indicated that over the course of therapy clients tended to have increases in dominance in their interpersonal patterns over the course of therapy (Filak et al., 1986; Sledge, 1999; Werner 1984). Thus, these findings provide indicate that TLIPT clients on average may tend to experience increases in dominance, as a result of therapy. Future research may explore if this finding differs for those clients who begin therapy with a highly rigid, dominant interpersonal pattern.

Both the therapist rating of the client on the IMI and the client self-rated IMI-GO consistently indicated changes in the hypothesized directions for interpersonal rigidity and extremity, affiliation, and control at the trend level or better. The therapist rating of the client on the IMI reflects the behavior of the client within the therapeutic relationship, while the IMI-GO may reflect a change in perception that indicates a change in a client's working model of self in interacting with others. Thus, the changes indicated by these distinct measures appear to reflect changes in different elements of clients' interpersonal operations.

Also notable is the lack of any significant interpersonal changes in observers' ratings of clients, while therapists and clients both identified significant interpersonal changes in clients' patterns. One explanation for this may be that interpersonal changes are most salient within the context of the therapeutic relationship. Furthermore, the 
therapist who is in direct contact with the client may be more prone to observe these changes at a "gut level”, or through more covert, nonverbal channels. The observer who does not participate in and is not present in the interaction, may not have the same level of experiencing with the client and therefore may not have the same sense of interpersonal change and may not be attuned to subtle differences in the feel of being with the client. Additionally, without direct interaction, observers may not have been able to register the changes that are measured by the IMI. The IMI is designed to measure the covert reactions of interactants and the instructions specify that the rater respond as if "in the presence" of the person being rated. Since observers did not directly interact with the clients, it may be that observers were not sensitive to changes in interpersonal patterns that are measured with the IMI. That is, observations of therapy tapes may not have provided observers as full of a sense of the clients' interpersonal style, as direct interaction would have.

Study Question Two: What Factors Contribute to Interpersonal Changes in Clients

\section{Treated with TLIPT?}

\section{Complementarity Reductions across Stages of TLIPT}

Analyses revealed a statistically significant reduction in affiliation complementarity from the first four sessions of therapy through the subsequent five sessions, although there was no evidence of a reduction in control complementarity. These findings are consistent with a stage model of TLIPT, where client and therapist dyads experience reductions in affiliation complementarity from the beginning to the middle phase of therapy. Other research has shown that complementarity in successful therapy follows a high-low-high pattern (Tracey et al., 1999). As suggested by 
interpersonal theory, these results support that the beginning stage of therapy may focus on building a strong and secure therapeutic relationship through elements, such as complementarity along the affiliation dimension. As therapeutic dyads enter the middle stage of therapy, the relationship becomes more unstable, thus less complementary. During this unstable phase, it may be that therapists are more prone to consciously disengage from complementarity to client's maladaptive interpersonal patterns. It may also be possible that as the relationship moves into the middle stage of therapy, clients also tend to reduce their complementary responses to the therapist, perhaps suggesting an increased comfort to disengage from the therapist.

\section{Variables Associated with Interpersonal Changes}

This study also provided some initial exploratory analyses of variables that may contribute to decreases in interpersonal rigidity and extremity, increases in affiliation, and increases in dominance. Comparing those with high interpersonal change and low interpersonal change on these variables helps to determine if these variables may predict interpersonal change for future research. Additionally, TLIPT therapists and other therapists may continue to develop focus on these variables in therapy to bring about interpersonal change in therapy clients. Contrary to hypotheses, there were no significant differences among any of the clusters on early working alliance, early symptom reductions, early affiliation complementarity, and middle stage control complementarity. However, several variables showed promise as predictors of interpersonal change that warrant further exploration in future research.

Those with large increases in dominance had higher early control complementarity than those with small to moderate reductions in dominance at the level 
of a trend. Additionally, those in the high reductions of rigidity and extremity cluster had higher early control complementarity than those with stable or small increases in rigidity and extremity at a level approaching a trend. Future research may specifically test the role of early control complementarity in bringing about reductions in rigidity and extremity and increases in dominance. As discussed earlier, TLIPT clients tended to be submissive at the beginning of therapy. Thus, typically high early control complementarity would involve the therapist taking a dominant stance. A dominant stance may be comfortable for TLIPT therapists, as research has indicated that therapists tend to have a friendly-dominant style (Celani, 1974; Coulter, 1993). Additionally, high control complementarity reflects the lack of a power struggle between client and therapist. The comfort for both therapists and clients by providing this complementary response may contribute to the development of a secure atmosphere for clients to subsequently attempt to broaden their range of interpersonal behaviors and thus reduce their rigidity and extremity.

Those with moderate to large increases in friendliness had higher later affiliation complementarity than those with some moderate reductions in friendliness at the level of a trend. This ran contrary to the hypothesis that lower complementarity in the middle phase will lead to greater interpersonal change. One explanation is that as has been found by Tracey et al. (1999) among others, more successful cases still have more complementarity in the middle phase than less successful cases. Thus, those with greater increases in affiliation may have reductions in complementarity, although they still have a higher level of complementarity than those with less therapy success (i.e., reductions in friendliness). Another explanation is that the clusters for affiliation change were not as 
clearly defined as anticipated. While individuals in cluster one had moderate to large increases in affiliation on all three measures (i.e., IMI-GO, significant other, and therapist ratings), the individuals in cluster two had mixed ratings. That is, cluster two individuals had moderate reductions in affiliation for therapist and significant other ratings, but also had moderate increases in affiliation on IMI-GO ratings. This inconsistency of the IMIGO ratings may be due to clients seeing their affiliation changes differently than outside others. As described earlier, clients' IMI-GO ratings were the only measure of the five that rated clients in the friendly range of affiliation at the beginning of therapy. It may be that clients have a tendency to see themselves as friendly and getting friendlier over the course of therapy, regardless of how others perceive them.

\section{The Role of Common Factors}

Through a review of research, Asay and Lambert (1999) have suggested four categories of factors common to all forms of psychotherapy that account for outcomes of therapy. These factors include extratherapeutic factors such as the significant events taking place in clients' lives, the therapeutic relationship, expectancy or hope that therapy will help, and the specific rationale provided by the theoretical approach. The design and measures used in this study cannot rule out the possibility that common factors contributed to the results. However, this pattern of results also suggests that factors specific to the TLIPT approach did contribute beyond common factors. TLIPT provided a rationale, guidance, and emphasis on the therapeutic relationship. The results followed a pattern that is highly consistent with what is predicted by the TLIPT theory. Further, the results were consistent across a variety of measures, completed by different raters, which assess variables specifically linked to TLIPT theory. 


\section{Clinical Implications of the Study}

Although by no means definitive, this study provides some potential guidance for therapists of interpersonal and other orientations, particularly in the area of interpersonal change in clients. Results support that interpersonal changes are an important outcome for interpersonal therapy, and for therapy in general. Therapists may utilize the TLIPT approach to foster clinically significant symptom reductions, reduction of interpersonal problems, increases in interpersonal affiliation and control, and decreases in interpersonal rigidity and extremity. One caution, however, is that increases in interpersonal affiliation and control may not be desirable for all clients. While it appears that clients in TLIPT generally appear to increase in affiliation, therapists should utilize the CMP conceptualization to determine specific interpersonal change targets for each client. Additionally, therapists from any orientation may benefit from increased awareness of interpersonal change as an outcome of therapy and those variables that may help to facilitate interpersonal change, such as variations in complementarity over the stages of psychotherapy.

Limitations of this Study, Strengths of this Study, and Future Research

A major limitation of this study is that it is not a randomized clinical trial and therefore lacks a control group and comparison group. This limits the conclusions that can be drawn from the results presented here. However, as described above, due to the stability of the inventories used to measure symptoms and interpersonal change, the likelihood that results are superfluous is greatly reduced. The changes that were measured in TLIPT clients were often of magnitudes great enough to indicate reliable changes that were unlikely to be caused by spontaneous recovery or regression toward 
the mean. This is consistent with other research that has shown that Brief Relational Therapy, which is similar to TLIPT, leads to clinically significant change, and this change is at least equal to cognitive-behavioral therapy and a greater change than that found in psychodynamic therapy (Muran, Safran, Samstag, \& Winston, in press; Safran, Muran, Samstag, \& Winston, in press).

Nevertheless, future research may further study TLIPT by subjecting it to a randomized clinical trial, comparing it with an alternative treatment and/or control group. This type of study would provide a definitive test of the hypothesis that TLIPT produces symptom reductions and interpersonal change outcomes beyond what might be expected in other forms of treatment and/or a non-treatment condition. In other words, this would provide a test of the effectiveness of TLIPT.

Further limitations of these analyses included the limited sample size and the conservative nature of the Bonferroni correction used for these analyses. These factors in combination may have limited the power to find small to moderate effects. The sample size placed limitations on the statistical techniques that could be applied to the data However, within the area of psychotherapy research this sample size of 61 represents a moderate sample size. As parts of this study were exploratory in nature, numerous comparisons were planned. In order to control for alpha inflation a Bonferroni correction was applied. While this may have decreased the power of the study, this helped avoid spurious results. Nonetheless, statistical trends were interpreted due to the exploratory nature of the study. Future research may utilize these trends to provide focus for further analysis of interpersonal change. 
Another limitation of this study was incomplete or missing data. Although data collection was based upon a schedule, at various times and for varying reasons some measures were not filled out by clients, therapists, observers, or the significant others. This led to differing numbers included in analyses. Analyses were able to accommodate the varying availability of data. While some measures such as significant other ratings had a great deal of missing data, analysis of these measures provided important insights.

A strength of this study was the multi-measure, multi-rater approach to measuring interpersonal change. This provided the opportunity to examine interpersonal change from a variety of perspectives and therefore provide a more complete view of interpersonal change as an outcome of TLIPT. Strengths of the multi-measure, multirater approach to the assessment of interpersonal pattern change include providing assessments in varying spheres of clients' lives. Research has preliminarily supported that repetitive interpersonal themes occur within clients' relationships (Luborsky et al., 1985), there is consistency of interpersonal themes across clients' narratives in psychotherapy (Crits-Christoph, Demorest, Muenz, \& Baranackie, 1994) and interpersonal themes are reenacted within the therapy relationship (Fried, CritsChristoph, \& Luborsky, 1992). However, the interpersonal style the client utilizes in the therapeutic relationship may not necessarily be indicative of the client's interpersonal style with others outside of therapy. This study attempted to address this area as it examined if changes occur in interpersonal patterns within the therapy relationship as perceived by the therapist and the observer, with a significant other relationship outside of therapy, and from clients' own perception of their interpersonal approach with others. One implication from this approach is to provide information about the raters and the 
measures that are more likely and less likely to perceive interpersonal change. For instance, therapists and clients were most consistent in recognizing interpersonal change in the client, while observers did not seem to perceive consistent interpersonal change. Further, while the IIP registered decreases in interpersonal distress, it did not show changes on other interpersonal measures.

As Connolly et al. (1999) indicate, it is likely that psychopathology is developed via multiple pathways. This study focused on a sample of clients selected and treated for interpersonal problems. Therefore, by selection, this study examined a sample of clients whose symptoms were largely attributed to maladaptive interpersonal patterns. Future research, building off of this study, may explore how important interpersonal changes are in maintaining symptom gains following therapy versus other potential etiological factors. That is, future research can test the hypothesis that individuals who experience interpersonal pattern changes in therapy are more likely to maintain low levels of symptoms after therapy, than those without interpersonal changes. Additionally, future research can further clarify the kinds of interpersonal change that may lead to the maintenance of symptom improvements. 


\section{Summary and Conclusions}

Overall, evidence emerged that TLIPT clients experienced reductions in symptoms that go beyond what would be expected from spontaneous remission, i.e. beyond the cut off scores and RCIs established for the OQ45. Additionally, TLIPT clients experienced changes on measures of interpersonal patterns, which have been shown to remain stable in individuals who are not in therapy. Thus, evidence supports that TLIPT contributes to both symptom reduction and interpersonal pattern change. Analyses also indicated that TLIPT therapist and client dyads experienced reductions in complementarity from the beginning to the middle phase of therapy. Additionally, preliminary evidence was found suggesting that future research may explore the role of early control complementarity in producing reductions in interpersonal rigidity and extremity and increases in interpersonal dominance. Future research may also explore the role of later affiliation complementarity in producing increases in interpersonal affiliation.

Examining interpersonal outcomes may be an important component particularly when looking at a therapy designed to bring about interpersonal changes. Connolly et al. (1996) and Connolly et al. (2000) reported that the interpersonal pattern that the client enacts with the therapist is also a prominent interpersonal style for the client outside of therapy in $44-60 \%$ of cases. Thus, while this indicates that the interpersonal patterns enacted in therapy are often utilized outside of therapy, this is not always the case. Thus, a multi-rater approach to measuring interpersonal patterns can provide important data for interpersonal changes beyond the therapeutic relationship. This study found preliminary evidence that clients' interpersonal patterns changed in the therapeutic relationship (i.e., 
as rated by therapists), changed in relationships outside of therapy (i.e., as rated by significant others), and changed in the clients' general approach to others (i.e., as rated by the IMI-GO).

Interpersonal change appears to be an important outcome of psychotherapy. Interpersonal change may occur alongside of symptom reduction, but appears to be distinct. Interpersonal change appears to represent a change in a more stable, trait-like pattern. Future research should continue to explore interpersonal change as an outcome of psychotherapy. 


\section{References}

Alden, L., Wiggins, J., \& Pincus, A. (1990). Construction of circumplex scales for the Inventory of Interpersonal Problems. Journal of Personality Assessment, 55, 521536.

Anderson, E., \& Lambert, M. (1995). Short-term dynamically oriented psychotherapy: A review and meta-analysis. Clinical Psychology Review, 19, 503-514.

Asay, T. P., \& Lambert, M. J. (1999). The empirical case for common factors in therapy: Quantitative findings. In M. A. Hubble, B. L. Duncan, \& S. D. Miller (Eds.), The heart and soul of change: What works in therapy (pp.23-55). Washington, DC: American Psychological Association.

Barber, J.P., Crits-Cristoph, P., \& Luborsky, L. (1992). Effects of therapist adherence and competence on patient outcome in brief dynamic therapy. Journal of Consulting and Clinical Psychology, 64, 619-622.

Beck, A., Ward, C., Mendelson, M., Mock, J., \& Erbaugh, J. (1961). An inventory for measuring depression. Archives of General Psychiatry, 41, 561-571.

Benjamin, L. S., Giat, L., \& Estroff, S. E. (1981). Manual for coding social interactions in terms of Structural Analysis of Social Behavior. Madison: University of Wisconsin.

Borkovec, T., Newman, M., Pincus, A., \& Lytel, R. (2002). A component analysis of cognitive-behavioral therapy for generalized anxiety disorder and the role of 
interpersonal problems. Journal of Consulting and Clinical Psychology, 70, 288298.

Bowlby, J. (1970). Disruption of affectional bonds and its effects on behavior. Journal of Contemporary Psychotherapy, 2, 75-86.

Bowlby, J. (1988). A secure base: Parent-child attachment and healthy human development. New York: Basic Books.

Brokaw, D. W. (1983). Markov chains and master therapists: An interpersonal analysis of psychotherapy process. Dissertation Abstracts International, 44, 1585-B.

Campbell, S. R., \& Brown, R. A. (1990, August). The relationship of interpersonal complementarity to marital satisfaction and security. Paper presented at the $98^{\text {th }}$ annual convention of the American Psychological Association, Boston, MA.

Carson, R. (1969). Interaction concepts of personality. Chicago: Aldine.

Casey, L. M., Oei, T. P. S., \& Newcombe, P. A. (2005). Looking beyond the negatives: A time period analysis of positive cognitions, negative cognitions, and working alliance in cognitive-behavior therapy for panic disorder. Psychotherapy Research, 15, 55-68.

Caspar, F., Grossmann, C., Unmussig, C., \& Schramm, E. (2005). Complementary therapeutic relationship: Therapist behavior, interpersonal patterns, and therapeutic effects. Psychotherapy Research, 15, 91-102.

Celani, D. P. (1974). The complementarity hypothesis: An exploratory study. Unpublished doctoral dissertation, University of Vermont, Montpelier.

Connolly, M. B., Crits-Christoph, P., Barber, J. P., \& Luborsky, L. (2000). Transference 
patterns in the therapeutic relationship in supportive-expressive psychotherapy for depression. Psychotherapy Research, 10, 356-372.

Connolly, M. B., Crits-Christoph, P., Demorest, A., Azarian, K., Muenz, L., \& Chittams, J. (1996). Varieties of transference patterns in psychotherapy. Journal of Consulting and Clinical Psychology, 64, 1213-1221.

Connolly, M. B., Crits-Christoph, P., Shelton, R. C., Hollon, S., Kurtz, J., Barber, J. P. et al. (1999). The reliability and validity of a measure of self-understanding of interpersonal patterns. Journal of Counseling Psychology, 46, 472-482.

Connolly Gibbons, M. B. (2004). The role of interpersonal relationships in the process of psychotherapy. Psychotherapy Research, 14, 401-414.

Constantino, M. J. (2003). Interpersonal/intrapsychic process in psychotherapy: The impact of self-verification strivings on the working alliance. Dissertation Abstracts International, 64 (01), 412B. (UMI No. AAI3076940)

Consumer Reports. (1995, November). Mental health: Does therapy help? pp. 734-739.

Coulter, L. P. (1993). Effects of therapist experience and interpersonal style on complementarity of responses. Unpublished doctoral dissertation, University of North Carolina, Chapel Hill.

Crits-Christoph, P. (1992). The efficacy of brief psychodynamic psychotherapy: A meta-analysis. American Journal of Psychiatry, 149, 151-158.

Crits-Cristoph, P., Demorest, A., \& Connolly, M. B. (1990). Quantitative assessment of interpersonal themes over the course of psychotherapy. Psychotherapy: Theory, Research, Practice, Training, 27, 513-521.

Crits-Christoph, P., Demorest, A., Muenz, L. R., \& Baranackie, K. (1994). Consistency 
of interpersonal themes for patients in psychotherapy. Journal of Personality, 62, 499-526.

Davies-Osterkamp, S., Strauss, B., \& Schmitz, N. (1996). Interpersonal problems as predictors of symptom related treatment outcome in long-term psychotherapy. Psychotherapy Research, 6, 164-176.

Derogatis, L. R. (1977). SCL-90: Administration, scoring, and procedures manual for the revised version. Baltimore: Clinical Psychometric Research.

Derogatis, L. R. (1983). Brief Symptom Inventory (BSI): Administration, scoring, and procedures manual. Minneapolis, MN: National Computer Systems.

Dietzel, C. S., \& Abeles, N. (1975). Client-therapist complementarity and therapeutic outcome. Journal of Counseling Psychology, 22, 264-272.

Elkin, I., Shea, T., Watkins, J. T., Imber, S. D., Sotsky, S. M., Collins, J. F. et al. (1989). National Institute of Mental Health treatment of depression collaborative research program: General effectiveness of treatments. Archives of General Psychiatry, 46, 971-982.

Estroff, S. D., \& Nowicki, S., Jr. (1992). Interpersonal complementarity, gender of interactants, and performance on puzzle and word tasks. Personality and Social Psychology Bulletin, 18, 351-356.

Filak, J., Abeles, N., \& Norquist S. (1986). Clients’ pretherapy interpersonal attitudes and psychotherapy outcome. Professional Psychology: Research and Practice, 17, 217-222.

Fried, D., Crits-Christoph, P., \& Luborsky, L. (1992). The first empirical demonstration 
of transference in psychotherapy. Journal of Nervous and Mental Disease, 180, 326-331.

Gaston, L. (1990). The concept of the alliance and its role in psychotherapy: Theoretical and empirical considerations. Psychotherapy, 27, 143-153.

Gaston, L., Marmar, C. R., Gallagher, D., \& Thompson, L. W. (1991). Alliance prediction of outcome beyond in-treatment symptomatic change as psychotherapy processes. Psychotherapy Research, 1, 104-112.

Granberg, A., \& Armelius, K. (2003). Change of self-image in patients with neurotic, borderline, and psychotic disturbances. Clinical Psychology and Psychotherapy, 10, 228-237.

Hartley, D. E., \& Strupp, H. H. (1983). The therapeutic alliance: Its relationship to outcome in brief psychotherapy. In J. Masling (Ed.), Empirical studies of analytic concepts (Vol. 1, pp. 1-37). Hillsdale, NJ: Analytic Press.

Henry, W. P., Schacht, T. E., \& Strupp, H. H. (1986). Patient and therapist introject, interpersonal process, and differential psychotherapy outcome. Journal of Consulting and Clinical Psychology, 54, 27-31.

Holm-Hadulla, R. (1995). Psychische Storunger von Sturdiervenden Habilitrationshscrift. Heidelberg, Germany: Medizinische Fakultat.

Horowitz, L. M., Locke, K. D., Morse, M. B., Walker, S. V., Dryer, D. C., Tarrow, E. et al. (1991). Self-derogations and the interpersonal theory. Journal of Personality and Social Psychology, 61, 68-79.

Horowitz, L., Rosenberg, S., Baer, B., Ureno, G., \& Villasenor, V. (1988). Inventory of Interpersonal Problems: Psychometric properties and clinical applications. 
Journal of Consulting and Clinical Psychology, 56, 885-892.

Horvath, A. O., \& Bedi, R. P. (2002). The alliance. In J. C. Norcross (Ed.), Psychotherapy relationships that work: Therapist contributions and responsiveness to patients. New York: Oxford University Press.

Horvath, A. O., \& Greenberg, L. S. (1986). The development of the Working Alliance Inventory. In W. M. Pinsof \& L. S. Greenberg (Eds.), The psychotherapeutic process: A research handbook. New York: Guilford Press.

Horvath, A. O., \& Greenberg, L. S. (1989). Development and validation of the Working Alliance Inventory. Journal of Counseling Psychology, 36, 223-233.

Horvath, A. O., \& Greenberg, L. S. (Eds.). (1994). The Working Alliance: Theory, Research, and Practice. New York: Wiley \& Sons.

Horvath, A. O., \& Luborsky, L. (1993). The role of the therapeutic alliance in psychotherapy. Journal of Consulting and Clinical Psychology, 61, 561-573.

Horvath, A. O., \& Symonds, B. (1991). Relation between working alliance and outcome in psychotherapy: A meta-analysis. Journal of Counseling Psychology, 36, 223233.

Howard, K. I., Kopta, S. M., Krause, M. S., \& Orlinsky, D. E. (1986). The dose-effect relationship in psychotherapy. American Psychologist, 41, 159-164.

Jacobson, N. S., \& Truax, P. (1991). Clinical significance: A statistical approach to defining meaningful change in psychotherapy research. Journal of Consulting and Clinical Psychology, 56, 885-892.

Kiesler, D. J. (1983). The 1982 Interpersonal Circle: A taxonomy for complementarity in human transactions. Psychological Review, 90, 185-214. 
Kiesler, D. J. (1987). Research manual for the Impact Message Inventory. Palo Alto, CA: Consulting Psychologist Press.

Kiesler, D. J. (1996). Contemporary interpersonal theory and research. New York: John Wiley.

Kiesler, D. J., \& Schmidt, J. (1993). The Impact Message Inventory: Form IIA Octant Scale Version. Palo Alto, CA: Mind Garden.

Kiesler, D. J., \& Watkins, L. M. (1989). Interpersonal complementarity and the therapeutic alliance: A study of relationship in psychotherapy. Psychotherapy, 26, $183-194$.

Kivlighan, D. M., McGovern, T. V., \& Corazzini, J. G. (1984). Effects of content and timing of structuring interventions on group therapy process and outcome. Journal of Counseling Psychology, 31, 363-370.

Kopta, S., Howard, K., Lowry, J., \& Beutler, L. (1994). Patterns of symptomatic recovery in psychotherapy. Journal of Consulting and Clinical Psychology, 62, 1009-1016.

Laird, H., \& Vande Kemp, H. (1987). Complementarity as a function of stage in therapy: An analysis of Minuchin’s structural family therapy. Journal of Marital and Family Therapy, 13, 127-137.

Lambert, M. (1992). Implications of outcome research for psychotherapy integration. In J. C. Norcross \& M. R. Goldstein (Eds.), Handbook of psychotherapy integration (pp.99-124). New York: Basic Books.

Lambert, M., Hansen, N., Umpress, V., Lunnen, K., Okiishi, J., Burlingame, G. et al. (1996). Administration and scoring manual for the OQ45.2. Stevenson, MD: 
America Professional Credentialing Services LLC.

Leary, T. (1957). Interpersonal diagnosis of personality. New York: Ronald.

Levenson, H. (1995). Time-limited dynamic psychotherapy. New York: Basic Books.

Luborsky, L. (1976). Helping alliances in psychotherapy. In J. L. Cleghhorn (Ed.), Successful psychotherapy (pp. 92-116). New York: Brunner/Mazel.

Luborsky, L., McLellan, A. T., Woody, G., O’Brien, C. P., \& Auerbach, A. (1985).

Therapist success and its determinants. Archives of General Psychiatry, 42, 602611.

Malcolm, W. M. (2000). Relating process to outcome in the resolution of unfinished business in process experiential psychotherapy. Dissertation Abstracts International, 60 (08), 4235B. (UMI No. AAINQ39287)

Martin, D. J., Garske, J., \& Davis, M. K. (2000). Relation of the therapeutic alliance with outcome and other variables: A meta-analytic review. Journal of Consulting and Clinical Psychology, 68, 438-450.

McCullough, J. P., \& Carr, K. F. (1987). Stage process design: A predictive confirmation structure for the single case. Psychotherapy, 24, 759-768.

McMain, S. F. (1996). Relating changes in self-other schemas to psychotherapy outcome. Dissertation Abstracts International, 56 (10), 5775B. (UMI No. AAMNN99634)

Murrell, S. (2003). Rationale for an “interactionist” interpersonal psychotherapy (Sixth Revision). Unpublished manuscript. Department of Psychological and Brain Sciences, University of Louisville, Louisville, KY 40208.

Murrell, S., Mehl, R., Buck, K., Nichols, K., \& Fearing, T. (1999). Evaluation of 
assumptions underlying a time-limited psychotherapy for interpersonal problems.

Poster presentation at the American Psychological Association Convention.

Boston, August, 1999.

Muran, J. C. (1993). The self in cognitive-behavioral research: An interpersonal perspective. The Behavior Therapist, 16, 69-73.

Muran, J., Safran, J., Samstag, L., Gorman, B., Twining, L., \& Winston, A. (1995). Linking in-session change to overall outcome in short-term cognitive therapy. Journal of Consulting and Clinical Psychology, 63, 651-657.

Muran, J. C., Safran, J. D., Samstag, L. W., \& Winston, A. (in press). Evaluating an alliance-focused treatment for personality disorders. Psychotherapy.

Muran, J. C., Samstag, L. W., Jilton, R., Batchelder, S., \& Winston, A. (1992a). Measuring patient-therapist interactions across time from a third-party perspective. Unpublished manuscript, Beth Israel Medical Center, New York, NY.

Muran, J. C., Samstag, L. W., Jilton, R., Batchelder, S., \& Winston, A. (1992b). Relation of interpersonal behavior and transactions to alliance and outcome over time in short-term psychotherapy. Unpublished manuscript, Beth Israel Medical Center, New York, NY.

Muran, J. C., Segal, Z. V., Samstag, L. W., \& Crawford, C. E. (1994). Patient pretreatment interpersonal problems and therapeutic alliance in short-term cognitive therapy. Journal of Consulting and Clinical Psychology, 62, 185- 190.

Nelson, A. P. (1984). Rigidity in the interpersonal functioning of psychiatric patients and normals. Dissertation Abstracts International, 44 (09), 2902 B. 
Nolan, S. A., Strassle, C. G., Roback, H. B., \& Binder, J. L. (2004). Negative treatment effects in dyadic psychotherapy: A focus on prevention and intervention strategies. Journal of Contemporary Psychotherapy, 34, 311-330.

Raymond, L., Friedlander, M. L., Heatherington, L., Ellis, M. V., \& Sargent, J. (1993). Communication processes in structural family therapy: Case study of an anorexic family. Journal of Family Psychology, 6, 308-326.

Rice, A. H. (2003). Interpersonal problems of persons with personality disorders and group outcomes. International Journal of Group Psychotherapy, 53, 155-175.

Rosenthal, R., Muran, J., Pinsker, H., Hellerstein, D., \& Winston A. (1999). Interpersonal change in brief supportive psychotherapy. Journal of Psychotherapy Research and Practice, 8, 55-63.

Rudy, J. P., McLemore, C. W., \& Gorsuch, R. L. (1985). Interpersonal behavior and therapeutic progress: Therapists and clients rate themselves and each other. Psychiatry, 48, 264-281.

Ruiz, M. A., Pincus, A. L., Borkovec, T. D., Echemendia, R. J., Castonguay, L. G., \& Ragusea, S. A. (2004). Validity of the Inventory of Interpersonal Problems for predicting treatment outcome: An investigation with the Pennsylvania Practice Research Network. Journal of Personality Assessment, 83, 213-222.

Safran, J. D., \& Muran, J. (2000). Negotiating the therapeutic alliance. New York: The Guilford Press.

Safran, J. D., Muran, J. C., Samstag, L. W., \& Winston, A. (in press). Evaluating an alliance-focused treatment for potential treatment failures. Psychotherapy.

Salsman, N. L., McGuffin, S., \& Murrell, S. A. (2002, August). Client progress over 
three stages of Time-Limited Interpersonal Therapy. Paper presented at the 2002 annual convention of the American Psychological Association, Chicago, IL.

Salsman, N. L., \& Murrell, S. A. (2003). Therapeutic alliance levels and outcomes in Time-Limited Interpersonal Therapy. Paper presented at the 2003 annual meeting of the North American Society for Psychotherapy Research, Newport, RI.

Salsman, N. L., Ulmer, C. S., \& Murrell, S. A. (2002). Time-Limited Interpersonal Therapy interventions and outcomes. Paper presented at the 2002 annual convention of the American Psychological Association, Chicago, IL.

Saltzman, C., Luetgert, M. J., Roth, C. H., Creaser, J., \& Howard, T. (1976). Formation of a therapeutic relationship: Experiences during the initial phase of psychotherapy as predictors of treatment duration and outcome. Journal of Consulting and Clinical Psychology, 44, 546-555.

Schacht, T., Binder, J., \& Strupp, H. (1984). The dynamic focus. In H. H. Strupp \& J. L. Binder, Psychotherapy in a new key: A guide to time-limited dynamic psychotherapy. New York: Basic Books.

Schauenburg, H., Kuda, M., Sammet, I., \& Strack, M. (2000). The influence of interpersonal problems and symptom severity on the duration and outcome of short-term psychodynamic psychotherapy. Psychotherapy Research, 10, 133146.

Schmidt, J. A., Wagner, C. C., \& Kiesler, D. J. (1999). Psychometric and circumplex properties of the octant scale Impact Message Inventory (IMI-C): A structural evaluation. Journal of Counseling Psychology, 46, 325-334.

Shapiro, D., Barkum, M., Rees, A., Hardy, G., Reynolds, S., \& Startup, M. (1994). 
Effects of treatment duration and severity of depression on the effectiveness of cognitive behavioral and psychodynamic-interpersonal psychotherapy. Journal of Consulting and Clinical Psychology, 62, 522-534.

Shields, C., Murrell, S. A., \& Salsman, N. (in press). Changes in the interpersonal characteristics of a client who "recovered" from anxiety and depression following interactionist interpersonal therapy. Clinical Case Studies.

Shim, H. S., \& Chung, N. W. (1998). Interpersonal complementarity and counseling outcome. Korean Journal of Counseling and Psychotherapy, 10, 95-119.

Simpson, J. A., \& Rholes, W. S. (1998). Attachment theory and close relationships. New York: Guilford Press.

Sledge, S. B. (1999). Treatment of chronic depression with ICBT-CD: A preliminary treatment-outcome study assessing interpersonal and attributional correlates of chronic depression. Dissertation Abstracts International, 59 (12), 6496B. (UMI No. AAM9915161)

Spielberger, C. D., Gorsuch, R. C., \& Lushene, R. E. (1970). Manual for the State Trait Anxiety Inventory. Palo Alto, CA: Consulting Psychologists Press.

Strong, S. R., Hills, H. I., Kilmartin, C. T., DeVries, H., Lanier, K., Nelson, B. N., et al. (1988). The dynamic relations among interpersonal behaviors: A test of complementarity and anticomplementarity. Journal of Personality and Social Psychology, 54, 798-810.

Strupp, H. H. (1980a). Success and failure in time limited psychotherapy: A systematic comparison of two cases (Comparison 1). Archives of General Psychiatry, 37, 595-603. 
Strupp, H. H. (1980b). Success and failure in time limited psychotherapy: A systematic comparison of two cases (Comparison 2). Archives of General Psychiatry, 37, 708-716.

Strupp, H. H. (1980c). Success and failure in time limited psychotherapy: Further evidence (Comparison 4). Archives of General Psychiatry, 37, 947-954.

Strupp, H. H. \& Binder, J. (Eds.). (1984). Psychotherapy in a new key: A guide to timelimited psychodynamic psychotherapy. New York: Basic Books.

Sullivan, H. S. (1953a). Conceptions of modern psychiatry. New York: Norton.

Sullivan, H. S. (1953b). The interpersonal theory of psychiatry. New York: Norton.

Svartberg, M., \& Stiles, T. (1992). Predicting patient change from therapist competence and patient-therapist complementarity in short-term anxiety-provoking psychotherapy: A pilot study. Journal of Consulting and Clinical Psychology, 60, 304-307.

Svartberg, M., Stiles, T. C., \& Seltzer, M. H. (2004). Randomized, controlled trial of the effectiveness of Short-Term Dynamic Psychotherapy and Cognitive Therapy for cluster c personality disorders. American Journal of Psychiatry, 161, 810-817.

Tasca, G. A. (1988). Complementarity in the psychotherapeutic relationship. Unpublished doctoral dissertation, University of Saskatehcewan, Saskatoon, Canada.

Tasca, G. A., \& McMullen, L. M. (1993). Interpersonal complementarity and antitheses within a stage model of psychotherapy. Psychotherapy, 29, 515-523.

Teyber, E. (2000). Interpersonal Process in Psychotherapy: A Relational Approach, Fourth Edition. Belmont, CA: Brooks/Cole. 
Tracey, T. J. (1986). Interactional correlates of premature termination. Journal of Consulting and Clinical Psychology, 54, 784-788.

Tracey, T. J. (1987). Stage differences in the dependencies of topic initiation and topic following behavior. Journal of Counseling Psychology, 34, 123-131.

Tracey, T. J. (1993). An interpersonal stage model of the therapeutic process. Journal of Counseling Psychology, 40, 1-14.

Tracey, T. J. (1994). An examination of the complementarity of interpersonal behavior. Journal of Personality and Social Psychology, 67, 864-878.

Tracey, T. J., \& Kokotovic, A. M. (1989). Factor structure of the Working Alliance Inventory. Psychological Assessment, 1, 207-210.

Tracey, T. J., \& Ray, P. B. (1984). Stages of successful time-limited counseling: An interactional examination. Journal of Counseling Psychology, 31, 13-27.

Tracey, T. J., Sherry, P., \& Albright, J. (1999). The interpersonal process of cognitivebehavioral therapy: An examination of complementarity over the course of treatment. Journal of Counseling Psychology, 46, 80-91.

Van Denburg, T. F., \& Kiesler, D. J. (1993). Transactional escalation in rigidity and intensity of interpersonal behaviour under stress. British Journal of Medical Psychology, 66, 15-31.

Vermeersch, D. A., Lambert, M. J., \& Burlingame, G. M. (2000). Outcome questionnaire: Item sensitivity to change. Journal of Personality Assessment, 74, 242-261.

Wagner, C. C. (1995). A constructivist test of interpersonal complementarity: A 
comparison of self and peer ratings of interpersonal behavior. Unpublished doctoral dissertation, Virginia Commonwealth University, Richmond.

Wagner, C. C., Kiesler, D., \& Schmidt, J. (1995). Assessing the interpersonal transaction cycle: Convergence of action and reaction interpersonal circumplex measures. Journal of Personality and Social Psychology, 69, 938-949.

Wagner, J. R. (2003). Evaluating a core battery: A comparison of standardized, individualized, and qualitative approaches to therapy outcome assessment. Dissertation Abstracts International, 63 (11), 5541B. (UMI No. AAI3071980)

Wampold, B. E. (2001). The great psychotherapy debate. New Jersey: Lawrence Erlbaum Associates.

Watson, J. C., \& Gellar, S. M. (2005). The relation among the relationship conditions, the working alliance, and outcome in both process-experiential and cognitivebehavioral psychotherapy. Psychotherapy Research, 15, 25-33.

Weinstock-Savoy, D. E. (1986). The relationship of therapist and patient interpersonal styles to outcome in brief dynamic psychotherapy. Dissertation Abstracts International, 47, 2638B.

Weissman, M. M., \& Bothwell, S. (1976). The assessment of social adjustment by patients self-report. Archives of general psychiatry, 33, 1111-1115.

Werner, M. (1984). Das Impact Message Inventory: Ein interpersonaler personlich keitsfragebogen von Donald J. Kiesler (1976). Uebersetzung aus dem Amerikanischen und erste validierungen. Unpublished doctoral dissertation, University of Bern, Switzerland.

Wettersten, K. B., Lichtenber, J. W., \& Mallinckrodt, B. (2005). Associations between 
working alliance and outcome in Solution-Focused Brief Therapy and brief interpersonal therapy. Psychotherapy Research, 15, 35-43.

Wiggins, J., Trapnell, P., \& Phillips, N. (1988). Psychometric and geometric characteristics of the revised interpersonal scales (IAS-R). Multivariate Behavioral Research, 23, 517-530.

Wilberg, T., Karterud, S., Pedersen, G., Urnes, O., Irion, T., Brabrand, J. et al. (2003). Outpatient group psychotherapy following day treatment for patients with personality disorders. Journal of Personality Disorders, 17, 510-521.

Wilbereg, T., Karterud, S., Urnes, O., Pedersen, G., \& Friis, S. (1998). Outcomes of poorly functioning patients with personality disorders in a day treatment program. Psychiatric Services, 49, 1462-1467.

Willett, J. (1988). Questions and answers in the measurement of change. In E. Rothkopf (Ed.), Review of research in education (1988-89) (pp. 345-422). Washington, DC: American Educational Research Association.

Woodward, L., Murrell, S., \& Bettler, B. (in press). Stability, reliability, and norms for the Inventory of Interpersonal Problems. Psychotherapy Research. 


\section{CURRICULUM VITAE}

NAME: $\quad$ Nicholas Lawrence Salsman

ADDRESS: Vanderbilt Community Mental Health Center

Suite 2200 Village at Vanderbilt

$150021^{\text {st }}$ Avenue South

Nashville, TN 37212

nicholas.l.salsman@vanderbilt.edu

DOB: $\quad$ Lexington, Kentucky - April 7, 1978

EDUCATION

\& TRAINING: $\quad$ B.A., Magna cum laude, with honors in Psychology

Transylvania University

1996-2000

M.A., Clinical Psychology

University of Louisville 2000-2003

Anticipated date to receive Ph.D., Clinical Psychology: 08/15/05

University of Louisville

Department of Psychological and Brain Sciences, Clinical 2000-2005

Clinical Psychology Internship

Vanderbilt University

2004-2005

AWARDS: Kentucky Psychological Association Hager Award

First Place Graduate Student Poster Competition

2004

University of Louisville Grawemeyer

Research Fellowship

2003 - 2004

North American Society for Psychotherapy Research

Student Research Travel Award

2003

Kentucky Psychological Association Hager Award

First Place Graduate Student Poster Competition

2003

Graduate of Future Professors Program

2002 


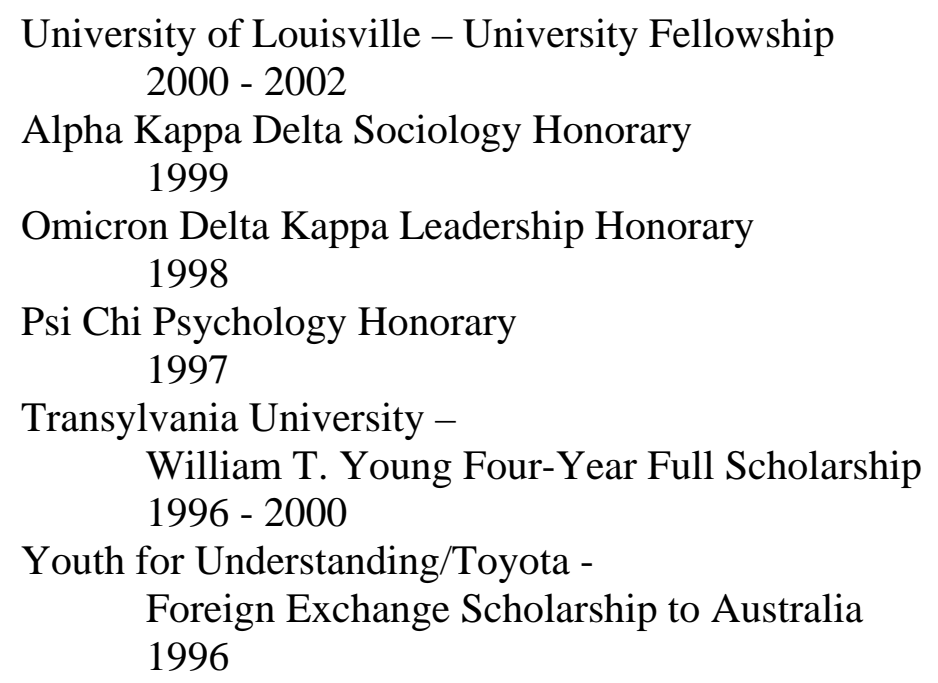

\section{PROFESSIONAL SOCIETIES:}

Association for the Advancement of Behavior Therapy, Student Affiliate 2005 - Present

Society for Psychotherapy Research, Student Affiliate 2003 - Present

Division of Psychotherapy, Division 29 of the APA, Student Affiliate 2002 - Present

American Psychological Association, Student Affiliate 1998 - Present

Kentucky Psychological Association, Student Affiliate 1998 - 2004

\section{PUBLICATIONS:}

Salsman, N. L., Murrell, S. A., \& Bettler, R. F. (2005). Are therapeutic alliance rupture resolution processes requisite, beneficial, or detrimental for positive therapeutic change? Manuscript submitted for publication.

Salsman, N. L., Merlo, L. J., \& Van Denburg, T. F. (2005). Alcohol expectancies mediate the relationship between attachment and alcohol consumption. Manuscript submitted for publication.

Shields, C., Murrell, S. A., \& Salsman, N. (in press). Changes in the interpersonal characteristics of a client who "recovered" from anxiety and depression following interactionist interpersonal therapy. Clinical Case Studies.

Murrell, S. A., Salsman, N. L., \& Meeks, S. (2004). Positive and negative psychological states and economic status as mediators of the relationship of education to fatigue among older adults. Research on Aging, 26, 673-700.

Murrell, S. A., Salsman, N. L., \& Meeks, S. (2003). Educational attainment, positive psychological mediators, and resources for health and vitality in older adults. Journal of Aging and Health, 15, 591-615. 


\section{NATIONAL MEETING PRESENTATIONS:}

Eells, T. D. \& Salsman, N. L. (2005, June). Problem identification and goal setting in psychotherapy: Comparing novice, experienced, and expert cognitive-behavioral and psychodynamic psychotherapists. Paper presented at the 2005 Society for Psychotherapy Research Conference, Montreal, Canada.

Salsman, N. L. \& Murrell, S. A. (2003, November). Therapeutic alliance levels and outcomes in Time-Limited Interpersonal Therapy. Poster presented at the 2003 North American Society for Psychotherapy Research Conference, Newport, RI and at the 2004 Kentucky Psychological Association Spring Academic Meeting.

Salsman, N. L., McGuffin, S., \& Murrell, S. A. (2002, August). Client progress over three stages of Time-Limited Interpersonal Therapy. Paper presented at the 2002 annual convention of the American Psychological Association, Chicago, IL.

Salsman, N. L., Ulmer, C., \& Murrell, S. A. (2002, August). Time-Limited Interpersonal Therapy interventions and outcome. Poster presented at the 2002 annual convention of the American Psychological Association, Chicago, IL and at the 2003 annual convention of the Kentucky Psychological Association, Louisville, KY.

McGuffin, S., Salsman, N. L., \& Murrell, S. A. (2001, August). The working alliance over three stages of Interpersonal Therapy. Poster presented at the 2001 annual convention of the American Psychological Association in San Francisco, CA.

Merlo, L. J., Salsman, N. L., \& Van Denburg, T. F. (2001, August). Underage drinking among the four adult attachment style categories. Poster presented at the 2001 annual convention of the American Psychological Association in San Francisco, CA.

Salsman, N. L., Merlo, L. J., \& Van Denburg, T. F. (2001, August). Alcohol expectancies within four groups of adult attachment styles. Poster presented at the 2001 annual convention of the American Psychological Association in San Francisco, CA. 